\title{
Extending Oblivious Transfer with Low Communication via Key-Homomorphic PRFs
}

\author{
Peter $\operatorname{Scholl}^{(\otimes)}(\mathbb{D}$ \\ Aarhus University, Aarhus, Denmark \\ peter.scholl@cs.au.dk
}

\begin{abstract}
We present a new approach to extending oblivious transfer with communication complexity that is logarithmic in the security parameter. Our method only makes black-box use of the underlying cryptographic primitives, and can achieve security against an active adversary with almost no overhead on top of passive security. This results in the first oblivious transfer protocol with sublinear communication and active security, which does not require any non-black-box use of cryptographic primitives.

Our main technique is a novel twist on the classic OT extension of Ishai et al. (Crypto 2003), using an additively key-homomorphic PRF to reduce interaction. We first use this to construct a protocol for a large batch of 1-out-of- $n$ OTs on random inputs, with amortized $o(1)$ communication. Converting these to 1-out-of-2 OTs on chosen strings requires logarithmic communication. The key-homomorphic PRF used in the protocol can be instantiated under the learning with errors assumption with exponential modulus-to-noise ratio.
\end{abstract}

\section{Introduction}

In an oblivious transfer protocol, a receiver wishes to learn a subset of some messages held by a sender, whilst hiding exactly which messages are received. A common type of oblivious transfer is 1-out-of-2 OT, where the sender holds messages $x_{0}, x_{1}$, while the receiver holds a bit $b$ and wishes to learn $x_{b}$. The protocol should guarantee that the receiver learns no information on $x_{1-b}$, whilst the sender learns nothing about $b$. 1-out-of-2 OT is a key tool in building secure two-party and multi-party computation protocols, and most efficient protocols need to use a very large number of oblivious transfers that scales with the input size [Yao86], or the size of the circuit description of the function being computed [GMW87].

All known protocols for oblivious transfer are much more expensive than standard symmetric-key primitives, as they rely on public-key cryptography. This property seems to be inherent, since it is known that constructing OT from symmetric cryptographic primitives in a black-box manner is impossible [IR89]. 
An essential technique for reducing the cost of oblivious transfer is OT extension, which reduces the cost of carrying out many OTs with amortization. OT extension protocols proceed in two stages: in a setup phase, a small number of 'seed' OTs are created using standard public-key techniques; secondly, these are extended to create many more, independent OTs, with a lower cost than the seed OT protocols. Typically the second phase is based only on cheap, symmetric cryptography, so using OT extension allows many OTs to be created with only $O(k)$ public-key operations for security parameter $k$, greatly reducing the computational costs.

OT Extension: A Brief History. Classically, OT extension refers to evaluating OT using mainly symmetric key cryptography. In this paper we broaden the term to cover any protocol that generates $m=$ poly $(k)$ OTs in a way which is more efficient than executing $m$ instances of an OT protocol. Reducing communication for the case of (1-out-of-2) bit-OT, where the sender's messages are bits, is of particular importance. This is exactly what is needed in the GMW protocol for secure multi-party computation [GMW87, Gol04], and a bit-OT protocol with $O(1)$ communication complexity implies secure computation with constant overhead using GMW, and even with active security [IPS08].

Beaver [Bea96] first showed how to convert $O(k)$ seed OTs into any polynomial number of OTs, using only one-way functions, for security parameter $k$. In this technique, the parties use a secure two-party computation protocol to evaluate the circuit that takes as input a random seed from each party, then applies a PRG and computes the OT functionality on the expanded, random inputs. With Yao's protocol [Yao86] this only needs $O(k)$ OTs, since the inputs are of size $O(k)$.

The 'IKNP' protocol, by Ishai et al. [IKNP03], lies at the core of all recent, practical OT extension protocols. IKNP was the first protocol to efficiently extend OT in a black-box manner, using only a hash function which satisfies a correlation robustness assumption (or a random oracle). The communication complexity of this protocol is $O(k+\ell)$ bits per extended OT with passive security, where $\ell$ is the bit length of the sender's strings. Harnik et al. [HIKN08] later showed how to obtain active security with the same asymptotic efficiency. The TinyOT protocol [NNOB12] introduced the first practical, actively secure OT extension, and more recently the overhead of active security has been reduced to almost nothing for both the 1-out-of-2 [ALSZ15, KOS15] and 1-out-of- $n$ cases [OOS17, PSS17].

These protocols are essentially optimal for transferring messages of length $\ell=\Omega(k)$, but when $\ell$ is short (as in bit-OT where $\ell=1$ ) there is still an overhead in $O(k)$. Kolesnikov and Kumaresan [KK13] presented a variant of the IKNP protocol based on 1-out-of- $n$ OT, which can be used to perform 1-outof-2 bit-OT with $O(k / \log k)$ communication. It is not known how to make this bit-OT protocol actively secure, because it relies on a passively secure reduction from 1-out-of- $n$ to 1-out-of-2 OT [NP99].

Unfortunately, all known methods for achieving constant-communication bit-OT use very complex techniques, and often require non-black-box use of 
cryptographic primitives. Ishai et al. [IKOS08] combined Beaver's non-black-box technique [Bea96] for OT extension with a polynomial-stretch PRG in NC0 and randomized encodings, to obtain a passively secure protocol with amortized $O(1)$ computational overhead (implying $O(1)$ communication). As well as needing a strong assumption on the PRG, a major drawback is the use of non-black-box techniques, which lead to a very high constant. The same authors later gave an alternative, black-box approach with constant communication [IKOS09]. However, this still requires heavy machinery such as algebraic geometry codes, randomized encodings and low-communication PIR. Additionally, achieving active security would require generic use of zero-knowledge proofs [GMW86, IKOS07], again with non-black-box use of the underlying primitives. Recently, Boyle et al. [BGI17] showed how to obtain an amortized communication cost of just 4 bits per bit-OT using homomorphic secret-sharing, which can be realised from either DDH [BGI16] or LWE [DHRW16]. As with the previous works, however, this construction makes non-black-box use of PRGs and would be extremely inefficient in practice.

Finally, we remark that using indistinguishability obfuscation and fully homomorphic encryption, it is possible to produce poly $(k)$ OTs on random inputs with a communication complexity that is independent of the number of OTs, with a general method for reusable correlated randomness in secure computation [HW15, HIJ+16].

\subsection{Contributions of This Work}

We present a new approach to extending oblivious transfer with low communication. Our protocol, in the random oracle model, makes black-box use of the underlying cryptographic primitives and can achieve security against an active adversary with almost no overhead on top of passive security. This results in the first bit-OT protocol with sublinear communication and active security, making only black-box use of cryptographic primitives. Table 1 compares the characteristics of our protocol with some of the other OT extension protocols discussed earlier.

Our main technique is a novel twist on the classic IKNP OT extension, using an additively key-homomorphic PRF to reduce interaction. The main challenge here is to handle the homomorphism error present in known key-homomorphic PRF constructions, without compromising on correctness or security. We first present a protocol for a large batch of 1-out-of- $p_{i}$ OTs on random inputs, for multiple, distinct primes $p_{i}$. The communication complexity of this protocol is sublinear in the total number of random OTs, with an amortized cost of $o(1)$ bits per OT. It was not known previously how to achieve this without using obfuscation, ${ }^{1}$ and this primitive may be useful in wider applications. If we want

\footnotetext{
${ }^{1}$ Even with obfuscation, secure computation with complexity sublinear in the output size and active security is known to be impossible [HW15]. The obfuscation-based protocol of $[\mathrm{HIJ}+16]$ circumvents this using a CRS, whilst we use the random oracle model.
} 
Table 1. Various protocols for extending 1-out-of-2 bit-OT (unless otherwise specified) with different assumptions. All passively secure protocols can be transformed to be actively secure using non-black-box zero-knowledge techniques. $\mathrm{CRH}$ is a correlationrobust hash function, RO is a random oracle; $\left(\begin{array}{l}n \\ 1\end{array}\right)$-ROT is 1-out-of- $n$ OT on random inputs.

\begin{tabular}{|c|c|c|c|c|}
\hline Protocol & $\begin{array}{l}\text { Communication } \\
\text { per OT (bits) }\end{array}$ & Security & Based on & Black-box \\
\hline [Bea96] & $\operatorname{poly}(k)$ & passive & OWF & $x$ \\
\hline [IKNP03] & $O(k)$ & passive & $\mathrm{CRH} / \mathrm{RO}$ & $\checkmark$ \\
\hline [KK13] & $O(k / \log k)$ & passive & $\mathrm{CRH} / \mathrm{RO}$ & $\checkmark$ \\
\hline [ALSZ15,KOS15] & $O(k)$ & active & $\mathrm{CRH} / \mathrm{RO}$ & $\checkmark$ \\
\hline [IKOS08] & $O(1)$ & passive & $\begin{array}{l}\text { poly-stretch } \\
\text { local PRG }\end{array}$ & $x$ \\
\hline [IKOS09] & $O(1)$ & passive & $\Phi$-hiding & $\checkmark$ \\
\hline [BGI17] & $4+o(1)$ & passive & $\mathrm{DDH}$ & $x$ \\
\hline$[\mathrm{HIJ}+16]\left(\left(\begin{array}{l}n \\
1\end{array}\right)-\mathrm{ROT}\right)$ & $o(1)$ & active & $\mathrm{iO}+\mathrm{FHE}$ & $x$ \\
\hline This work $\left(\left(\begin{array}{c}p_{i} \\
1\end{array}\right)-\mathrm{ROT}^{a}\right)$ & $o(1)$ & active & $\mathrm{LWE}+\mathrm{RO}$ & $\checkmark$ \\
\hline This work $\left(\left(\begin{array}{l}2 \\
1\end{array}\right)\right.$-OT $)$ & $O(\log k)$ & active & $\mathrm{LWE}+\mathrm{RO}$ & $\checkmark$ \\
\hline
\end{tabular}

to obtain 1-out-of-2 OT on chosen strings, each 1-out-of- $p_{i}$ random OT can be converted to a 1-out-of-2 OT with $O\left(\log p_{i}\right)$ bits of communication using standard techniques, giving logarithmic communication overhead.

The additively key-homomorphic PRF needed in our protocol can be instantiated based on the learning with errors assumption with an exponential modulusto-noise ratio. This assumption has previously been used to construct attributebased encryption [GVW13] and fully key-homomorphic encryption [BGG+14], and is believed to be hard if the LWE dimension is chosen large enough to thwart known attacks. The downside of our approach is that this spectrum of LWE parameters results in fairly heavy computational costs for the parties, so it seems that the main uses of our protocol would be in low bandwidth environments where communication is much more expensive than computation.

As a contribution of independent interest, to implement the base OTs in our protocol we generalise the consistency check used for OT extension by Asharov et al. [ALSZ15], and adapt it for producing correlated OTs over any abelian group $\mathbb{G}$, instead of just XOR correlations over bit strings. These are 1-out-of-2 OTs where the sender's messages are all guaranteed to be of the form $\left(x_{i}, x_{i}+\Delta\right)$, for some fixed correlation $\Delta \in \mathbb{G}$. We also identify a crucial security flaw in the original protocol of Asharov et al. [ALSZ15, ALSZ17a], which leaks information on the receiver's inputs to a passively corrupted sender. After reporting this to the authors, their protocol has been modified to fix this [ALSZ17b], and we use the same fix for our protocol. 


\subsection{Overview of Techniques}

IKNP OT Extension. We first recall the IKNP OT extension protocol [IKNP03] (with optimizations from [ALSZ13, KK13]), which uses $k$ instances of oblivious transfer to construct $m=\operatorname{poly}(k)$ oblivious transfers with only a cryptographic hash function and a pseudorandom generator. The parties begin by performing $k$ 1-out-of-2 OTs on random $k$-bit strings with their roles reversed. The receiver acts as sender in the base OTs, with input pairs of strings $\left(k_{i}^{0}, k_{i}^{1}\right)$. The sender, acting as receiver, inputs a random choice bit $s_{i}$ to the $i$-th OT and learns $k_{i}^{s_{i}}$, for $i=1, \ldots, k$. The receiver then sends over the values

$$
u_{i}=G\left(k_{i}^{0}\right) \oplus G\left(k_{i}^{1}\right) \oplus x
$$

where $G:\{0,1\}^{k} \rightarrow\{0,1\}^{m}$ is a pseudorandom generator (PRG) and $x=$ $\left(x_{1}, \ldots, x_{m}\right)$ are the receiver's $m$ choice bits. After this step, the parties can obtain $k$ correlated OTs on pairs of $m$-bit strings of the form $\left(t_{i}, t_{i} \oplus x\right)$, where $t_{i}=G\left(k_{i}^{0}\right)$ : the receiver knows both $t_{i}$ and $t_{i} \oplus x$, while the sender can define

$$
\begin{aligned}
q_{i} & =G\left(k_{i}^{s_{i}}\right) \oplus s_{i} \cdot u_{i} \\
& =t_{i} \oplus s_{i} \cdot x \\
& = \begin{cases}t_{i} & \text { if } s_{i}=0 \\
t_{i} \oplus x & \text { if } s_{i}=1\end{cases}
\end{aligned}
$$

This is a 1-out-of-2 OT on $m$-bit strings because the other message, $t_{i} \oplus \overline{s_{i}} \cdot x$, is computationally hidden to the sender due to the use of a PRG.

Both parties then place these values into matrices $Q, T \in\{0,1\}^{k \times m}$ contain$\operatorname{ing} q_{i}$ and $t_{i}$ (respectively) as rows, where the sender holds $Q$ and the receiver holds $T$. If $q^{j}, t^{j} \in\{0,1\}^{k}$ are the columns of $Q$ and $T$, and $s=\left(s_{1}, \ldots, s_{k}\right)$, then notice that we have

$$
t^{j}=q^{j} \oplus\left(x_{j} \cdot s\right)
$$

So, by transposing the matrix of OTs we obtain $m$ sets of correlated OTs on $k$-bit strings, with $x_{j}$ as the receiver's choice bits. Finally, the two parties can convert these correlated OTs into OTs on random strings using a hash function $H$ that satisfies a notion of correlation robustness (or, modeled as a random oracle): the sender computes the two strings $H\left(q^{j}\right)$ and $H\left(q^{j} \oplus s\right)$, whilst the receiver can only compute one of these with $H\left(t^{j}\right)$; the other string remains unknown to the receiver since it does not know $s$. This means the parties have converted $k$ initial OTs into $m=$ poly $(k)$ OTs on random strings, and these random OTs can be used to transfer the sender's chosen messages by encrypting them with a one-time pad.

Apart from the initial base OTs, the only interaction in this process is sending the $u_{i}$ values at the beginning, which costs $O(m k)$ bits of communication. This gives an overhead of $O(k)$ when the sender's inputs are bit strings of constant size. 
Using a Key-Homomorphic PRF. We observe that if the PRG, G, in the above protocol satisfies $G(x \oplus y)=G(x) \oplus G(y)$, and the base OTs are correlated so that $k_{i}^{1}=k_{i}^{0} \oplus r$ for some fixed string $r$, then the main step of interaction in IKNP can be removed. The homomorphic property of the PRG preserves the correlation, so the parties can obtain the OTs $\left(t_{i}, t_{i} \oplus x\right)$ (for random choice bits $x$ ) without any message from the receiver: the sender simply defines $q_{i}=G\left(k_{i}^{s_{i}}\right)$ while the receiver defines $t_{i}=G\left(k_{i}^{0}\right)$ and $x=G(r)$. We then have

$$
q_{i}=G\left(k_{i}^{s_{i}}\right)=G\left(k_{i}^{0} \oplus\left(s_{i} \cdot r\right)\right)=G\left(k_{i}^{0}\right) \oplus\left(s_{i} \cdot G(r)\right)=t_{i} \oplus s_{i} \cdot x,
$$

as previously.

Unfortunately, such XOR-homomorphic PRGs are not known to exist. Instead, we do know how to build almost-seed-homomorphic PRGs (and almostkey-homomorphic PRFs) $G: \mathbb{Z}_{q}^{n} \rightarrow \mathbb{Z}_{p}$, which satisfy

$$
G(x+y)=G(x)+G(y)+e \quad(\bmod p)
$$

where $q>p$ and $|e| \leq 1$ is an error term, based on the learning with rounding (LWR) or learning with errors (LWE) assumption [BLMR13, BP14]. We remark that it is possible to build an error-free key-homomorphic PRF in the random oracle model based on the decisional Diffie-Hellman assumption, with the simple construction $F(k, x)=H(x)^{k}$ [BLMR13]. However, here the output homomorphism is multiplicative instead of additive, which is more difficult to exploit in constructing OT extension.

Trying to apply these additively homomorphic PRGs (or PRFs) to the IKNP protocol brings about two main challenges. Firstly, since the homomorphism maps into $\mathbb{Z}_{p}$ and not $\mathbb{F}_{2}^{k}$, we obtain matrices $Q$ and $T$ containing $\mathbb{Z}_{p}$ elements instead of bits, which means there is no natural way of 'transposing' the OT matrix whilst preserving the correlated OT property. Secondly, the homomorphism error means that all of the OTs will be incorrect with high probability.

To handle the first problem, we choose $p$ to be a primorial modulus which is the product of $\ell$ primes, and then decompose the correlated OTs via the Chinese Remainder Theorem. ${ }^{2}$ This gives us an alternative to transposing the bit-matrix in IKNP; however, it means that instead of constructing 1-out-of-2 OT, we end up with 1-out-of- $p_{i}$ random OTs, for each prime factor $p_{i}$ in the modulus. The second problem of eliminating the error is more difficult. We observe that the receiver can always compute a homomorphism error $e$, such that the resulting error in the OT is given by $e^{\prime}=e \cdot s_{i}$, where $s_{i}$ is one of the sender's choice bits in the base OTs. It seems tempting to let the receiver just send over $e$ so that the sender can correct the error, but this may not be secure: each error leaks information about the unknown PRG key, and a large number of errors could leak information on the secret PRG outputs. To securely correct the error, the receiver instead samples some uniform noise $u$, which is used to mask $e$. To ensure that both parties still obtain the correct result, we must obliviously

\footnotetext{
${ }^{2}$ Ball et al. used a primorial modulus for a different application of constructing arithmetic garbled circuits [BMR16].
} 
transfer the masked error $u+s_{i} \cdot e^{\prime}$ to the sender. Since $s_{i}$ is a choice bit in the original base OTs, this can be done by without any additional OTs, by extending the base OTs once more with a (standard) PRG.

This last error-correction step introduces some interaction into the basic OT extension protocol, which is otherwise non-interactive. Importantly, the amount of data that needs sending only depends on the security parameter, and not the modulus. Since each distinct prime factor in the modulus produces one additional OT in the OT extension phase, choosing a sufficiently large modulus allows us to obtain an amortized communication cost of $o(1)$ bits per random OT. If we wish to construct 1-out-of-2 OTs, each random 1-out-of $p_{i}$ OTs can be converted to a single 1-out-of-2 OT with $\log p_{i}=O(\log k)$ bits of communication (see Appendix A).

Active security. To obtain active security, the above protocol needs to be modified in two ways. Firstly, we need to ensure that the correlation in the base OTs is created correctly, and secondly, we need to ensure that a malicious receiver does not cheat in the error-correction stage, which would cause incorrect outputs.

We first consider the error-correction step. A common technique for dealing with this is to compute random linear combinations of all the correlated OTs, then open the result [KOS15] and check correctness. However, this only achieves negligible cheating probability when the correlation is over a large field. In our case we use a ring with many zero divisors, and this method cannot be applied in general. Nevertheless, our situation is slightly different because the size of the adversarial deviations can be bounded by some value $B$ that is much smaller than the modulus. This means that for some error $d<B$ introduced by a cheating receiver, and random challenge $r \leftarrow \mathbb{Z}_{p}$, the product $d r \bmod p$ is statistically close to uniform in $\mathbb{Z}_{p}$ (for arbitrary $p$ ), which suffices to prove security when taking random linear combinations.

For the base OTs, the receiver can cheat in an arbitrary way when creating the correlations, which means the above check is not enough to prevent deviations here. It might be tempting to work around this problem by choosing a prime modulus $q$ for the base OTs (before applying the PRG/PRF to convert mod $p)$. The problem here is that we then wouldn't be able to transpose the base OTs, which is necessary for checking consistency via random linear combinations. Instead, we adopt a different approach used for OT extension in [ALSZ15], where the receiver sends hashes of every pair of base OTs, which are then checked for consistency by the sender. We show that this approach still works to prove that the OTs are correlated over an arbitrary abelian group, instead of just XOR correlations over bit strings. If the receiver cheats, they may guess a few bits of the sender's secret choice bits. This does not cause a problem for the OT extension phase, and we model this possibility in our setup functionality.

Instantiation based on DDH. It is possible to modify the above protocol to use a key-homomorphic PRF in the random oracle model based on the decisional 
Diffie-Hellman assumption, instead of using LWE or LWR. This has the advantage of avoiding the problems with homomorphism error, since the DDH-based PRF $F(k, x)=H(x)^{k}$ is noiseless. However, the drawback is that this protocol produces random 1-out-of- $p$ OTs, where $p$ is the order of a group in which DDH is hard. Since DDH is not hard if $p$ has small factors, these cannot be decomposed into smaller random OTs using the CRT, so this does not lead to any improvements to 1-out-of-2 OT extension. Nevertheless, random 1-out-of- $p$ OT for exponentially large $p$ (sometimes referred to as batch related-key oblivious PRF evaluation) can be used to construct private equality test and private set intersection protocols [PSZ18, KKRT16, OOS17], so this variation could be useful in these applications to reduce interaction at the cost of requiring exponentiations instead of only symmetric-key operations. More details on this protocol are given in the full version of this work.

\section{Preliminaries}

\subsection{Universally Composable Security}

We present ideal functionalities and security proofs in the universal composability framework [Can01], and assume some familiarity with this.

Informally speaking, for a protocol $\Pi$ which implements a functionality $\mathcal{F}$ in the $\mathcal{G}$-hybrid model, we let $\operatorname{HYBRID}_{\Pi, \mathcal{A}, \mathcal{Z}}^{\mathcal{G}}$ denote the view of an environment $\mathcal{Z}$ in an execution of the real protocol with the adversary $\mathcal{A}$ controlling the corrupted parties, in a hybrid model where all parties have access to the ideal functionality $\mathcal{G}$. We let $\operatorname{IDEA} \mathrm{L}_{\mathcal{F}, \mathcal{S}, \mathcal{Z}}$ denote the view of $\mathcal{Z}$ in the ideal execution, where the simulator $\mathcal{S}$ plays the role of the corrupted parties in $\Pi$ and interacts with the functionality $\mathcal{F}$. When the context is clear, we sometimes abbreviate the two executions by HYBRID and IDEAL.

We say that the protocol $\Pi$ securely realises the functionality $\mathcal{F}$ in the $\mathcal{G}$ hybrid model, if for every adversary $\mathcal{A}$ there exists a simulator $\mathcal{S}$, such that for every environment $\mathcal{Z}$,

$$
\operatorname{HYBRID}_{\Pi, \mathcal{A}, \mathcal{Z}}^{\mathcal{G}} \stackrel{\mathrm{c}}{\approx} \operatorname{IDEAL}_{\mathcal{F}, \mathcal{S}, \mathcal{Z}}
$$

where $\stackrel{\mathrm{c}}{\approx}$ is the standard notion of computational indistinguishability.

As well as the standard, computational security parameter $k$, we often use a statistical security parameter $\lambda$. This means that the advantage of any probabilistic poly $(k)$-time environment in distinguishing the two executions is at most $\operatorname{negl}(k)+O\left(2^{-\lambda}\right)$.

\subsection{Key-Homomorphic Pseudorandom Functions}

We now recall the definitions of additively key-homomorphic pseudorandom functions [BLMR13,BP14], and discuss the distribution of the homomorphism error in LWE-based constructions. Let $n, p$ and $q>p$ be integers. 
Definition 1 (Key-homomorphic PRF). A function $F: \mathbb{Z}_{q}^{n} \times\{0,1\}^{\ell} \rightarrow \mathbb{Z}_{p}$ is a key-homomorphic PRF if it is a PRF, and for all $k_{1}, k_{2} \in \mathbb{Z}_{q}$ and $x \in\{0,1\}^{\ell}$ it holds that:

$$
F\left(k_{1}+k_{2}, x\right)=F\left(k_{1}, x\right)+F\left(k_{2}, x\right) \in \mathbb{Z}_{p}
$$

We do not know of any PRFs satisfying the above property, where the homomorphism is additive over both the inputs and the outputs, so instead use the following, weaker definition.

Definition 2 (Almost key-homomorphic PRF). A function $F: \mathbb{Z}_{q}^{n} \times$ $\{0,1\}^{\ell} \rightarrow \mathbb{Z}_{p}$ is an almost key-homomorphic PRF if it is a PRF, and for all $k_{1}, k_{2} \in \mathbb{Z}_{q}$ and $x \in\{0,1\}^{\ell}$ it holds that:

$$
F\left(k_{1}+k_{2}, x\right)=F\left(k_{1}, x\right)+F\left(k_{2}, x\right)+e \in \mathbb{Z}_{p}
$$

where $|e| \leq 1$.

To realise this, we use the rounding function

$$
\lfloor x\rceil_{p}=\lfloor x \cdot(p / q)\rceil
$$

which scales $x \in \mathbb{Z}_{q}$ to lie in the interval $[0, p)$ and then rounds to the nearest integer. We now define the learning with rounding assumption [BPR12].

Definition 3 (Learning with Rounding). Let $n \geq 1$ and $q \geq p \geq 2$ be integers. For a vector $\boldsymbol{s} \in \mathbb{Z}_{q}^{n}$, define the distribution $\mathrm{LWR}_{s}$ to be the distribution over $\mathbb{Z}_{q}^{n}, \mathbb{Z}_{p}$ obtained by sampling $\boldsymbol{a} \leftarrow \mathbb{Z}_{q}^{n}$ uniformly at random, and outputting $\left(\boldsymbol{a}, b=\lfloor\langle\boldsymbol{a}, \boldsymbol{s}\rangle\rceil_{p}\right)$.

The decisional- $\mathrm{LWR}_{n, q, p}$ problem is to distinguish any desired number of samples $\left(\boldsymbol{a}_{i}, b_{i}\right) \leftarrow \mathrm{LWR}_{s}$ from the same number of samples taken from the uniform distribution on $\left(\mathbb{Z}_{q}^{n}, \mathbb{Z}_{p}\right)$, where the secret $s$ is uniform in $\mathbb{Z}_{q}^{n}$.

With this we can easily construct a key-homomorphic PRF in the random oracle model, with the function $F: \mathbb{Z}_{q}^{n} \times\{0,1\}^{\ell} \rightarrow \mathbb{Z}_{p}$ defined by

$$
F(k, x)=\lfloor\langle k, H(x)\rangle\rceil_{p}
$$

where $H:\{0,1\}^{\ell} \rightarrow \mathbb{Z}_{q}^{n}$ is a random oracle. This is an almost-key-homomorphic $\mathrm{PRF}$ under the $\mathrm{LWR}_{n, q, p}$ assumption [BPR12].

There are also constructions in the standard model based on learning with rounding or learning with errors [BLMR13,BP14]. All these constructions inherit the same error term, which comes from first computing a function that is linear in the key $k$, and then rounding the result. 
Can we Remove the Homomorphism Error? Applications such as distributed PRFs can work around the error term by suitably rounding the output [BLMR13]. However, in some applications, particularly those in this work, it would be very useful to have a noise-free PRF satisfying Definition 1. Two previous works $[\mathrm{BV} 15, \mathrm{BFP}+15]$ claimed that their LWE-based constructions can achieve a slightly weaker notion, where it is computationally hard for an adversary to come up with a query $x$ that violates key-homomorphism. Unfortunately, these claims are not correct, ${ }^{3}$ and it seems difficult to modify these PRFs to satisfy this.

To see why the error seems to be inherent in these PRFs, consider an experiment where we sample random values $r_{1}, r_{2} \leftarrow \mathbb{Z}_{q}$, and then test whether $\left\lfloor r_{1}+r_{2}\right\rceil_{p}=\left\lfloor r_{1}\right\rceil_{p}+\left\lfloor r_{2}\right\rceil_{p}$. This gives us the same result as testing for homomorphism error in $F$, since $H$ is a random oracle and the two keys are random. ${ }^{4}$ Let $x_{1}=\left\lfloor r_{1}\right\rceil_{p}, x_{2}=\left\lfloor r_{2}\right\rceil_{p}$ and define the relevant fractional components $e_{1}=r_{1} \cdot p / q$ $(\bmod 1), e_{2}=r_{2} \cdot p / q(\bmod 1)$, where the reduction modulo 1 is mapped to the interval $[-1 / 2,1 / 2)$. If $p$ divides $q$ then it holds that $e_{1}, e_{2}$ are uniformly random in the set $[-1 / 2,1 / 2) \cap(p / q) \mathbb{Z}$.

If there is no homomorphism error then we have

$$
\left\lfloor e_{1}+e_{2}\right\rceil=\left\lfloor e_{1}\right\rceil+\left\lfloor e_{2}\right\rceil
$$

Clearly when $e_{1} \geq 0$ there will be no error as long as $e_{2} \leq 0$. Similarly, when $e_{2} \geq 0$ and $e_{1} \leq 0$ there is no error. These two error-free possibilities cover approximately half of the space of possible choices of $\left(e_{1}, e_{2}\right) \in[-1 / 2,1 / 2)^{2}$. For the remaining cases, if we condition on $e_{1} \geq 0$ and $e_{2}>0$ then there will be an error whenever $e_{1}+e_{2} \geq 1 / 2$, which happens with probability around $1 / 2$. Symmetrically, in the remaining case of $e_{2} \geq 0$ and $e_{1}>0$ the error probability is around $1 / 2$, and combining these cases we get an overall error probability of approximately $1 / 4$. The exact error rate depends on whether $p$ divides $q$ and if $q / p$ is even or not, but is nevertheless always close to $1 / 4$.

\section{OT Extension Protocol}

We now describe our main protocol for extending oblivious transfer.

\subsection{Setup Functionality}

We use the setup functionality $\mathcal{F}_{\Delta \text {-ROT }}$, shown in Fig. 1 , to implement the base OTs in our main protocol. This functionality creates $k$ random, correlated OTs over an abelian group $\mathbb{G}$ (in our protocol we instantiate this with $\mathbb{G}=\mathbb{Z}_{q}$ ) where

\footnotetext{
${ }^{3}$ We have confirmed this through personal communication with an author of $[\mathrm{BFP}+15]$. This does not affect the main results of that work or [BV15].

4 This method also applies to known standard model KH-PRFs based on LWE, as these constructions all have the form $F(k, x)=\left\lfloor L_{x}(k)\right\rceil_{p}$ for some linear function $L_{x}: \mathbb{Z}_{q}^{n} \rightarrow \mathbb{Z}_{q}$.
} 
the sender inputs $\Delta \in \mathbb{G}^{n}$ and obtains messages of the form $\left(b_{i}, b_{i}+\Delta\right)$ for randomly sampled $b_{i} \in \mathbb{G}^{n}$. The receiver inputs the choice bits $s_{i} \in\{0,1\}$ in a setup phase, and during the correlated OT phase it learns $a_{i}$, which is either $b_{i}$ or $b_{i}+\Delta$, depending on $s_{i}$. This $\Delta$-OT stage of the functionality also allows a corrupt sender to attempt to guess (a subset of) the bits $s_{i}$, but if the guess fails then the functionality aborts. This leakage is necessary so that we can efficiently implement $\mathcal{F}_{\Delta \text {-ROT }}$, using the protocol we give in Sect. 4 .

$\mathcal{F}_{\Delta \text {-ROT }}$ also includes a Chosen OTs command, which further extends the base OTs on chosen (but not necessarily correlated) inputs from the sender, using the same choice bits from the receiver.

\section{Functionality $\mathcal{F}_{\Delta-\text { ROT }}^{n, k, \mathbb{G}}$}

Parameters: $n$, the length; $k$, the number of OTs; $\mathbb{G}$, a finite, additive abelian group.

Initialize: On receiving ( sid, init, $s_{1}, \ldots, s_{k}$ ) from $P_{A}$, where $s_{i} \in\{0,1\}$, and (sid, init) from $P_{B}$, store $\left\{s_{i}\right\}_{i}$ and ignore any subsequent (sid, init) messages.

$\Delta$-OTs: $\quad$ On input (sid, correlated, $\Delta$ ) from $P_{B}$, where $\Delta \in \mathbb{G}^{n}$, send (sid, correlated) to $P_{A}$ and do the following.

1. Sample $b_{i} \leftarrow \mathbb{G}^{n}$, for $i \in[k]$. If $P_{B}$ is corrupted, instead receive $b_{i}$ from $\mathcal{A}$.

2. Compute $a_{i}=b_{i}+s_{i} \cdot \Delta$

3. If $P_{A}$ is corrupted, receive $a_{i} \in \mathbb{G}^{n}$ from $\mathcal{A}$ and recompute $b_{i}=a_{i}-s_{i} \cdot \Delta$.

4. If $P_{B}$ is corrupted, wait for $\mathcal{A}$ to input a message (guess, $S$ ), where $S$ efficiently describes a subset of $\{0,1\}^{k}$. If $\left(s_{1}, \ldots, s_{k}\right) \in S$ then send (success) to $\mathcal{A}$. Otherwise, send $\perp$ to both parties and terminate.

5. Output $a_{i}$ to $P_{A}$ and $b_{i}$ to $P_{B}$.

Chosen OTs: On input (sid, chosen, $\left.B,\left(u_{i}^{0}, u_{i}^{1}\right)_{i=1}^{k}\right)$ from $\quad P_{B}$, and (sid, chosen, $B)$ from $P_{A}$, where $u_{i}^{0}, u_{i}^{1} \in[0, B-1]$, send (sid, chosen, $\left.\left(u_{i}^{s_{i}}\right)_{i=1}^{k}\right)$ to $P_{A}$.

Fig. 1. Extended correlated random oblivious transfer functionality over a group $\mathbb{G}$

\section{$3.2 \quad$ Random OT Protocol}

The functionality we implement is shown in Fig. 2. This produces a batch of $m \cdot \ell$ random OTs at once, consisting of $m$ sets of random 1-out-of- $p_{i}$ OTs, for each $i=1, \ldots, \ell$, where $p_{i}$ is the $i$-th prime and $\ell$ is a parameter of the protocol. Let $P_{\ell}=2 \cdot 3 \cdot 5 \cdots p_{\ell}$ be the product of the first $\ell$ primes.

The protocol, shown in Fig. 3 , starts with a setup phase where the parties

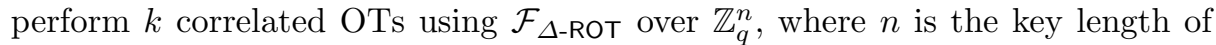
the almost-key-homomorphic PRF $F: \mathbb{Z}_{q}^{n} \times\{0,1\}^{k} \rightarrow \mathbb{Z}_{P_{\ell}}$. After this phase, $P_{R}$ holds random values $\boldsymbol{r}, \boldsymbol{b}_{i} \in \mathbb{Z}_{q}^{n}$, whilst $P_{S}$ holds random $s_{i} \in\{0,1\}$ and $\boldsymbol{a}_{i}=\boldsymbol{b}_{i}+s_{i} \cdot \boldsymbol{r}$, for $i=1, \ldots, k$.

In the OT extension phase, the parties expand the base OTs using $F$, such that the key-homomorphic property of $F$ preserves the correlation between $\boldsymbol{a}$ and $\boldsymbol{b}$, except for a small additive error. We have: 


\section{Functionality $\mathcal{F}_{p_{i} \text {-ROT }}^{m, \ell, k}$}

After receiving a message (send) from $P_{S}$ and (receive) from $P_{R}$, ignore all other messages from both parties and do as follows:

1. Sample choices $c_{1}^{i}, \ldots, c_{m}^{i} \leftarrow \mathbb{Z}_{p_{i}}$, for $i \in[\ell]$. If $P_{R}$ is corrupted, instead receive $c_{j}^{i} \in \mathbb{Z}_{p_{i}}$ from $\mathcal{A}$.

2. For each $j \in[m]$, sample $\sum_{i} p_{i}$ sets of strings $\left(y_{j}^{i, 0}, \ldots, y_{j}^{i, p_{i}-1}\right)_{i=1}^{\ell}$, where each $y_{j}^{i, c} \leftarrow\{0,1\}^{k}$.

3. Output $\left(y_{j}^{i, 0}, \ldots, y_{j}^{i, p_{i}-1}\right)$ to $P_{S}$ and $\left(c_{j}^{i}, y_{j}^{i, c_{i}^{j}}\right)$ to $P_{R}$, for $i \in[\ell]$ and $j \in[m]$.

Fig. 2. Functionality for $m$ sets of random $\left\{1 \text {-out-of- } p_{i}\right\}_{i=1}^{\ell}$ OTs on $k$-bit strings

$$
F\left(\boldsymbol{a}_{i}, j\right)=F\left(\boldsymbol{b}_{i}+s_{i} \cdot \boldsymbol{r}, j\right)=F\left(\boldsymbol{b}_{i}, j\right)+s_{i} \cdot\left(F(\boldsymbol{r}, j)+e_{i}\right)
$$

where $\left|e_{i}\right| \leq 1$ (note that $e_{i}$ depends on $j$, but we often omit the subscript- $j$ to simplify notation).

Since $P_{R}$ knows both $\boldsymbol{b}_{i}$ and $\boldsymbol{r}$, it can compute $e_{i}$, and then use the base OTs to obliviously transfer either $u_{i}+e_{i}$ (if $s_{i}=1$ ) or $u_{i}$ (if $s_{i}=1$ ) to $P_{S}$, where $u_{i}$ is a uniformly random value in $\{0, \ldots, B-1\}$, and $B$ is superpolynomial in the security parameter so that $u_{i}$ statistically masks $e_{i}$.

After step 2f, if $u_{i}+e_{i} \notin\{-1, B\}$ then we have:

$$
\begin{aligned}
q_{i}=q_{i}^{\prime}-v_{i} & =t_{i}^{\prime}+s_{i} \cdot\left(x_{j}+e_{i}\right)-u_{i}-s_{i} \cdot e_{i} \\
& =t_{i}^{\prime}-u_{i}+s_{i} \cdot x_{j} \\
& =t_{i}+s_{i} \cdot x_{j} \quad\left(\bmod P_{\ell}\right)
\end{aligned}
$$

For each $j \in[m+1]$, these $k$ sets of correlated OTs are then placed into vectors $\boldsymbol{q}_{j}$ and $\boldsymbol{t}_{j}$, which satisfy $\boldsymbol{q}_{j}=\boldsymbol{t}_{j}+x_{j} \cdot \boldsymbol{s}$. To convert these into random 1-out-of- $p_{i}$ OTs, for $i=1, \ldots, \ell$, each $\boldsymbol{t}_{j}$ is reduced modulo $p_{i}$ and then hashed with the random oracle to produce the receiver's output string. The $c$-th output of the sender, for $c \in\left\{0, \ldots, p_{i}-1\right\}$, in the $(i, j)$-th OT is defined as:

$$
H\left(\boldsymbol{q}_{j}-c \cdot s \bmod p_{i}\right)=H\left(\boldsymbol{t}_{j}+\left(x_{j}-c\right) \cdot s \bmod p_{i}\right)
$$

which for $c=x_{j}$ is equal to the receiver's output, as required. The sender's other outputs are computationally hidden to the receiver, since to compute them it would have to query $\boldsymbol{q}_{j}-x^{\prime} \cdot \boldsymbol{s} \bmod p_{i}$ to the random oracle for some $x^{\prime} \neq x_{j}$, but this requires guessing $\boldsymbol{s}$.

The only opportunity for a malicious receiver to misbehave in the protocol is when sending the $\left(u_{i}, u_{i}+e_{i}\right)$ values to the base OT functionality, to correct the errors. The consistency check phase prevents this, by opening a random linear combination of the correlated OTs and checking that the linear relation still holds. We must then discard the $(m+1)$-th set of OTs so that the opened values do not reveal anything about the receiver's outputs. This check allows a corrupt 


\section{Protocol $\Pi_{p_{i} \text {-ROT }}^{m, \ell, k}$}

Let $F: \mathbb{Z}_{q}^{n} \times\{0,1\}^{k} \rightarrow \mathbb{Z}_{P_{\ell}}$ be an almost-key-homomorphic PRF.

Let $H:\{0,1\}^{*} \rightarrow\{0,1\}^{k}$ be a random oracle and $G:\{0,1\}^{k} \rightarrow \mathbb{Z}_{P_{\ell}}^{m}$ be a PRG.

1. Setup phase.

(a) $P_{S}$ samples $s_{1}, \ldots, s_{k} \leftarrow\{0,1\}$, and $P_{R}$ samples $r \leftarrow \mathbb{Z}_{q}^{n}$

(b) Both parties initialize $\mathcal{F}_{\Delta \text {-ROT }}^{n, k, \mathbb{Z}_{q}}$, where $P_{S}$ inputs $\left(s_{1}, \ldots, s_{k}\right) . P_{R}$ then calls the functionality again with input (correlated, $\boldsymbol{r}$ ).

(c) $P_{R}$ receives $\boldsymbol{b}_{1}, \ldots, \boldsymbol{b}_{k}$ and $P_{S}$ receives $\boldsymbol{a}_{1}, \ldots, \boldsymbol{a}_{k}$ such that $\boldsymbol{a}_{i}=\boldsymbol{b}_{i}+s_{i} \cdot \boldsymbol{r}$.

2. Extension phase. ${ }^{a} P_{S}$ initializes a zero matrix $\mathbf{Q} \in \mathbb{Z}_{P_{\ell}}^{k \times(m+1)} . P_{R}$ initializes a zero matrix $\mathbf{T} \in \mathbb{Z}_{P_{\ell}}^{k \times(m+1)}$, and a vector $\boldsymbol{x} \in \mathbb{Z}_{P_{\ell}}^{m+1}$.

For each $j \in[m+1]$ :

(a) $P_{S}$ computes $q_{i}^{\prime}=F\left(\boldsymbol{a}_{i}, j\right) \in \mathbb{Z}_{P_{\ell}}$, for $i \in[k]$

(b) $P_{R}$ computes $t_{i}^{\prime}=F\left(\boldsymbol{b}_{i}, j\right), x_{j}=F(\boldsymbol{r}, j) \in \mathbb{Z}_{P_{\ell}}$

(c) $P_{R}$ computes the errors

$$
e_{i}=F\left(\boldsymbol{b}_{i}+\boldsymbol{r}, j\right)-t_{i}^{\prime}-x_{j} \in\{0,1\}, \quad \text { for } i \in[k]
$$

and samples $u_{i} \leftarrow[0, B-1]$

(d) $P_{R}$ calls $\mathcal{F}_{\Delta \text {-ROT }}^{n, k, \mathbb{Z}_{q}}$ on input (chosen, $B,\left(u_{i}, u_{i}+e_{i} \bmod B\right)$ )

(e) $P_{S}$ receives $v_{i}=u_{i}+s_{i} \cdot e_{i} \bmod B$ from $\mathcal{F}_{\Delta \text {-ROT }}^{n, k, \mathbb{Z}_{q}}$

(f) $P_{S}$ defines $q_{i}=q_{i}^{\prime}-v_{i}$, and $P_{R}$ defines $t_{i}=t_{i}^{\prime}-u_{i}$

It should now hold that

$$
q_{i}=t_{i}+s_{i} \cdot x_{j} \bmod P_{\ell}
$$

(g) $P_{S}$ stores $\boldsymbol{q}_{j}=\left(q_{1}, \ldots, q_{k}\right)$ in column $j$ of the matrix $\mathbf{Q}$.

(h) $P_{R}$ stores $\boldsymbol{t}_{j}=\left(t_{1}, \ldots, t_{k}\right)$ in column $j$ of $\mathbf{T}$, and $x_{j}$ in entry $j$ of the vector $\boldsymbol{x}$.

3. Consistency check.

(a) $P_{S}$ samples a seed $\sigma \leftarrow\{0,1\}^{k}$ and sends this to $P_{R}$

(b) Both parties compute $\boldsymbol{\alpha}=(G(\sigma) \| 1)$.

(c) $P_{R}$ computes and sends

$$
\tilde{\boldsymbol{t}}=\mathbf{T} \boldsymbol{\alpha}, \quad \tilde{x}=\boldsymbol{x}^{\top} \boldsymbol{\alpha}
$$

(d) $P_{S}$ checks that $\mathbf{Q} \boldsymbol{\alpha}=\tilde{\boldsymbol{t}}+\tilde{x} \boldsymbol{s}$

4. Output: $P_{S}$ samples a seed $\rho \leftarrow\{0,1\}^{k}$ and sends this to $P_{R}$. The parties then compute their outputs as follows:

- $P_{S}$ outputs, for $j \in[m]$ and $i \in[\ell]$ :

$H\left(i, \rho, \boldsymbol{q}_{j}^{i}\right), H\left(i, \rho, \boldsymbol{q}_{j}^{i}-\boldsymbol{s}\right), \ldots, H\left(i, \rho, \boldsymbol{q}_{j}^{i}-\left(p_{i}-1\right) \cdot \boldsymbol{s}\right), \quad$ where $\boldsymbol{q}_{j}^{i}=\boldsymbol{q}_{j} \bmod p_{i}$

- $P_{R}$ outputs, for $j \in[m]$ and $i \in[\ell]$ :

$$
x_{j}^{i}, H\left(i, \rho, \boldsymbol{t}_{j}^{i}\right), \quad \text { where } x_{j}^{i}=x_{j} \bmod p_{i}, \quad \boldsymbol{t}_{j}^{i}=\boldsymbol{t}_{j} \bmod p_{i}
$$

${ }^{a}$ Steps $2-4$ can be iterated by maintaining $j$ as a counter.

Fig. 3. Random 1-out-of- $p_{i}$ OT extension protocol 
receiver to attempt to guess a few of the $s_{i}$ bits by cheating in only a few OT instances. However, this is exactly the same behaviour that is already allowed

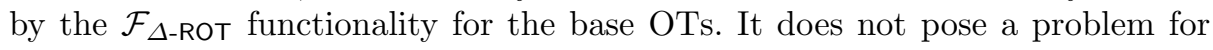
security because in the output phase $s$ is put through a random oracle, and the whole of $s$ must be guessed to break security.

\subsection{Security}

Theorem 1. Let $B=\Theta\left(2^{\lambda}\right), \ell=\Omega(k \lambda), F$ be an almost key-homomorphic PRF and $H$ be a random oracle. Then protocol $\Pi_{p_{i} \text {-ROT }}^{m, \ell, k}$ securely realises the functionality $\mathcal{F}_{p_{i} \text {-ROT }}^{m, \ell, k}$ with static security in the $\mathcal{F}_{\Delta \text {-ROT }}^{n, k, \mathbb{Z}_{q}}$-hybrid model.

We prove this by considering separately the cases of a corrupt sender and a corrupt receiver. Security when both parties are honest, or both corrupt, is straightforward.

Corrupt sender. This is the simpler of the two cases. We construct an idealworld simulator, $\mathcal{S}_{S}$, shown in Fig. 4 . The simulator uses random values to simulate the $v_{i}$ messages sent to $P_{S}$ during the OT extension phase, then samples $\tilde{x}$ at random to respond to the consistency check, computing $\tilde{\boldsymbol{t}}$ such that the check will always pass. The random oracle queries are responded to using knowledge of the sender's bits $s$ from the setup phase, so as to be consistent with the random

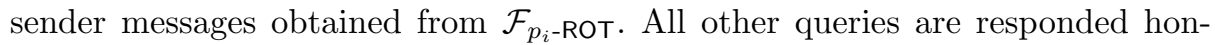
estly, at random. The security of the protocol against a corrupt sender rests on two key points: (1) $B=\Theta\left(2^{\lambda}\right)$, so that $u_{i}+e_{i}$ statistically masks the errors $e_{i}$ in the protocol, and (2) The security of the key-homomorphic PRF, which implies the $x_{j}$ outputs of the honest receiver are pseudorandom, and also the simulated $\tilde{x}$ is indistinguishable from the real value in the protocol.

Lemma 1. For every adversary $\mathcal{A}$ who corrupts $P_{S}$, and for every environment $\mathcal{Z}$, it holds that

$$
\operatorname{IDEAL}_{\mathcal{F}_{p_{i} \text {-ROT }}, \mathcal{S}_{S}, \mathcal{Z}} \stackrel{\mathrm{c}}{\approx} \operatorname{HYBRID}_{\Pi_{p_{i} \text { ROT }}, \mathcal{A}, \mathcal{Z}}^{\mathcal{F}_{\Delta-\mathrm{ZOT}}}
$$

Proof. Recall that as well as seeing the view of $\mathcal{A}$ during the protocol execution, the environment $\mathcal{Z}$ learns the outputs of both parties. We prove security by defining a sequence of hybrid executions, where $\mathrm{H}_{0}$ is defined to be the ideal process and each successive hybrid modifies the previous execution in some way.

Hybrid $\mathrm{H}_{1}$ : Instead of sampling $v_{i}$ at random, $\mathcal{S}_{S}$ sends $v_{i}=u_{i}+s_{i} \cdot e_{i} \bmod B$, where $u_{i} \leftarrow[0, B-1]$ and $e_{i}$ is computed as in the protocol, using randomly sampled $\boldsymbol{r} \in \mathbb{Z}_{q}^{n}$ and $\boldsymbol{b}_{i}:=\boldsymbol{a}_{i}-s_{i} \cdot \boldsymbol{r}$.

Hybrid $\mathrm{H}_{2}$ : Instead of sampling $\tilde{x}$ at random, let $x_{1}, \ldots, x_{m}$ be the outputs of the honest receiver (from $\mathcal{F}_{p_{i} \text {-ROT }}$ ), and sample $x_{m+1} \leftarrow \mathbb{Z}_{P_{\ell}}$. $\mathcal{S}_{S}$ then sends $\tilde{x}=\boldsymbol{x}^{\top} \boldsymbol{\alpha}$. 


\section{Simulator $\mathcal{S}_{S}$}

1. Setup phase. $\mathcal{S}_{S}$ receives $\boldsymbol{s}=\left(s_{1}, \ldots, s_{k}\right) \in\{0,1\}^{k}$ and $\boldsymbol{a}_{1}, \ldots, \boldsymbol{a}_{k} \in \mathbb{Z}_{q}^{n}$ from the corrupt sender, as input to $\mathcal{F}_{\Delta \text {-ROT }}^{n, k, \mathbb{Z}_{q}}$.

2. Extension phase.

(a) For each $j \in[m+1]$ and $i \in[k]$, whenever $\mathcal{S}_{S}$ receives a message (chosen, $B$ ) from $\mathcal{A}$ (as input to $\mathcal{F}_{\Delta \text {-ROT }}^{n, k, \mathbb{Z}_{q}}$ ), sample a random value $v_{i, j} \in[0, B-1]$ and return this to $\mathcal{A}$.

(b) Compute the matrix $\mathbf{Q}$ as $P_{S}$ should in the protocol, using knowledge of $\boldsymbol{a}_{i}$ and $v_{i, j}$.

(c) Call the functionality $\mathcal{F}_{p_{i} \text {-ROT }}$ to obtain the sender's outputs $\boldsymbol{y}_{j}^{i}=$ $\left(y_{j}^{i}[1], \ldots, y_{j}^{i}\left[p_{i}\right]\right)$, for $i \in[\ell]$ and $j \in[m]$.

3. Consistency check. After receiving the seed $\sigma$ from $\mathcal{A}$, compute $\boldsymbol{\alpha}=(G(\sigma) \| 1)$, then send $\tilde{x} \leftarrow \mathbb{Z}_{P_{\ell}}$ and $\tilde{\boldsymbol{t}}=\mathbf{Q} \boldsymbol{\alpha}-\hat{x} \boldsymbol{s}$ to $\mathcal{A}$.

4. Output/Random Oracle Queries. Receive the seed $\rho$ from $\mathcal{A}$, and whenever a query of the form $\left(i, \rho, \boldsymbol{q}_{j}^{i}-c \cdot s\right)$ is sent to the random oracle $H$, for $c \in\left[0, p_{i}-1\right]$, respond with $y_{j}^{i}[c]$.

Fig. 4. Simulator for a corrupted sender

Hybrid $\mathrm{H}_{3}$ : This is defined the same as $\mathrm{H}_{2}$, except the random choices $x_{1}, \ldots, x_{m+1}$ are replaced with values computed from the key-homomorphic PRF as $x_{j}=F(\boldsymbol{r}, j)$, using the previously sampled $\boldsymbol{r}$.

Note that all of the simulated messages in the final hybrid, $\mathrm{H}_{3}$, are identically distributed to messages sent in the real execution, and the outputs of the sender and receiver are computed exactly as in the protocol as outputs of the random oracle $H$ and PRF $F$, respectively. Therefore, $\mathrm{H}_{3} \equiv$ HYBRID.

Hybrids $\mathrm{H}_{0}$ and $\mathrm{H}_{1}$ are identically distributed as long as $u_{i}+s_{i} \cdot e_{i} \notin\{-1, B\}$, since $e_{i} \in\{0, \pm 1\}$. If the reduction modulo $B$ overflows then $\mathcal{Z}$ could distinguish because the outputs in $\mathrm{H}_{1}$ will be incorrect. This occurs with probability at most $2 / B$ for each $i$, so when $B=\Omega\left(2^{\lambda}\right)$ we have $\mathrm{H}_{0} \stackrel{\text { s }}{\approx} \mathrm{H}_{1}$.

In hybrid $\mathrm{H}_{2}$, the value $\tilde{x}$ is masked by $x_{m+1}$ so is uniformly random in the view of $\mathcal{Z}$. Therefore, $\mathrm{H}_{1} \equiv \mathrm{H}_{2}$.

Hybrids $\mathrm{H}_{2}$ and $\mathrm{H}_{3}$ are computationally indistinguishable, by a standard reduction to the key-homomorphic PRF, since $\boldsymbol{r}$ is uniformly random and not seen by the environment, so the $x_{j}$ values are pseudorandom. This completes the claim that IDEAL $\stackrel{\mathrm{c}}{\approx}$ HYBRID.

Corrupt receiver. The simulator $\mathcal{S}_{R}$, given in Fig. 5, essentially runs an internal copy of the sender and honestly generates messages as $P_{S}$ would. The main challenge is to extract the inputs of the corrupt $P_{R}$, and also show that $\mathcal{Z}$ cannot query the random oracle $H$ on a value corresponding to one of the sender's random outputs that was not chosen by the receiver. 


\section{Simulator $\mathcal{S}_{R}$}

1. Setup phase. $\mathcal{S}_{R}$ receives $\boldsymbol{r}$ and $\boldsymbol{b}_{1}, \ldots, \boldsymbol{b}_{k}$ from the corrupt receiver, as input to $\mathcal{F}_{\Delta \text {-ROT }}^{n, k, \mathbb{Z}_{q}}$. It then samples $\boldsymbol{s} \leftarrow\{0,1\}^{k}$ and defines $\boldsymbol{a}_{i}=\boldsymbol{b}_{i}+s_{i} \boldsymbol{r}$.

For any key query guess submitted by $\mathcal{A}$ to $\mathcal{F}_{\Delta \text {-ROT }}^{n, k, \mathbb{Z}_{q}}, \mathcal{S}$ responds according to the secret $\boldsymbol{s}$. If any guess is unsuccessful, $\mathcal{S}$ aborts.

2. Extension phase.

(a) $\mathcal{S}$ waits for $P_{R}$ to send a message of the form (chosen, $B, u_{i}, u_{i}^{\prime}$ ) as input to $\mathcal{F}_{\Delta \text {-ROT. }}^{n, k, \mathbb{Z}_{q}} \mathcal{S}$ then defines $e_{i}^{\prime}=u_{i}^{\prime}-u_{i} \bmod B$ and $v_{i}=u_{i}+s_{i} \cdot e_{i}^{\prime}$.

(b) $\mathcal{S}$ computes $q_{i}$ as an honest sender would, and defines the matrix $\mathbf{Q}$.

3. Consistency Check.

(a) $\mathcal{S}$ samples $\sigma \leftarrow\{0,1\}^{k}$ and sends this to $P_{R}$.

(b) $\mathcal{S}$ receives $\tilde{\boldsymbol{t}}, \tilde{x}$ and checks that $\mathbf{Q} \boldsymbol{\alpha}=\tilde{\boldsymbol{t}}+\tilde{x} \boldsymbol{s}$. If the check fails, abort.

(c) Extract the inputs $x_{j}^{\prime}$ using Proposition 1.

(d) Call $\mathcal{F}_{p_{i} \text {-ROT }}$ and send the choices $\left\{x_{j}^{\prime} \bmod p_{i}\right\}_{i \in[\ell]}$, for $j \in[m]$. Receive back the OT outputs $y_{j}^{i}$, for $i \in[\ell], j \in[m]$.

4. Output phase/Random Oracle Queries. $\mathcal{S}$ sends a random seed $\rho \leftarrow\{0,1\}^{k}$, and responds to the random oracle queries as follows:

(a) If a query is $\left(i, \rho,\left(\boldsymbol{q}_{j}-x_{j}^{\prime} \boldsymbol{s}\right) \bmod p_{i}\right)$ for some $i \in[\ell], j \in[m]$ then respond with $y_{j}^{i}$. Otherwise, respond at random (consistent with previous queries).

Fig. 5. Simulator for a corrupted receiver

We use the following technical lemma to analyse the soundness of the consistency check when taking random linear combinations over the ring $\mathbb{Z}_{P_{\ell}}$.

Lemma 2. Let $\mathbf{E} \in \mathbb{Z}_{P_{\ell}}^{k \times(m+1)}$. Suppose that every column $\boldsymbol{e}_{i}$ of $\mathbf{E}$ satisfies $\left\|\boldsymbol{e}_{i}\right\|_{\infty} \leq B$, and further that there is at least one column not in $\operatorname{span}(\mathbf{1})$. Then,

$$
\underset{\alpha \leftarrow \mathbb{Z}_{P_{\ell}}^{m} \times\{1\}}{\operatorname{Pr}}[\mathbf{E} \boldsymbol{\alpha} \in \operatorname{span}(\mathbf{1})] \leq 2 B / P_{\ell}
$$

Proof. From the assumption that at least one column of $\mathbf{E}$ not in $\operatorname{span}(\mathbf{1})$, there exist two rows $\boldsymbol{a}, \boldsymbol{b}$ of $\mathbf{E}$ with $\boldsymbol{a} \neq \boldsymbol{b}$. If $\mathbf{E} \boldsymbol{\alpha} \in \operatorname{span}(\mathbf{1})$ then $\langle\boldsymbol{a}, \boldsymbol{\alpha}\rangle=\langle\boldsymbol{b}, \boldsymbol{\alpha}\rangle$ and so $\langle\boldsymbol{a}-\boldsymbol{b}, \boldsymbol{\alpha}\rangle=0$. Let $\boldsymbol{d}=\boldsymbol{a}-\boldsymbol{b}$, and $j$ be an index where $d_{j} \neq 0$. Then $\langle\boldsymbol{d}, \boldsymbol{\alpha}\rangle=0$ if and only if $d_{j} \alpha_{j}=-\sum_{i \neq j} d_{i} \alpha_{i}$.

First consider the case that $j \neq m+1$, so $\alpha_{j}$ is uniform in $\mathbb{Z}_{P_{\ell}}$. For any fixed choice of $d_{j}$, the number of distinct possibilities for $d_{j} \alpha_{j} \bmod P_{\ell}$ is given by the order of $d_{j}$ in the additive group $\mathbb{Z}_{P_{\ell}}$, which equals $P_{\ell} / \operatorname{gcd}\left(d_{j}, P_{\ell}\right)$. Since $\left|a_{j}\right|,\left|b_{j}\right| \leq B$, we have $d_{j} \in[0,2 B] \cup\left[P_{\ell}-2 B, P_{\ell}-1\right]$, from which it follows that $\operatorname{gcd}\left(d_{j}, P_{\ell}\right) \leq 2 B$. Therefore, since $\alpha_{j}$ is random and independent of $\sum_{i \neq j} \alpha_{i} d_{i}$, we have that $\operatorname{Pr}[\langle\boldsymbol{d}, \boldsymbol{\alpha}\rangle=0] \leq 2 B / P_{\ell}$.

On the other hand, if $j=m+1$ then $\alpha_{j}=1$. But this means $d_{j}=$ $-\sum_{i \neq j} d_{i} \alpha_{i}$, and because $d_{j} \neq 0$ there must be another index $j^{\prime}$ with $d_{j^{\prime}} \neq 0$. We then apply the previous argument on $j^{\prime}$ to obtain the same probability.

If $\boldsymbol{\alpha}$ is sampled using a PRG with a public seed, instead of uniformly at random, the previous statement still holds except with negligible probability. 
Lemma 3. Let $\mathbf{E}$ be as in Lemma 2 and let $G:\{0,1\}^{k} \rightarrow \mathbb{Z}_{P_{\ell}}^{m} \times\{1\}$ be a PRG. Then,

$$
\operatorname{Pr}_{\sigma \leftarrow\{0,1\}^{k}}[\mathbf{E} \boldsymbol{\alpha} \in \operatorname{span}(\mathbf{1}): \boldsymbol{\alpha}=G(\sigma)] \leq 2 B / P_{\ell}+\operatorname{negl}(k)
$$

Proof. Define a distinguisher, $D$, for the PRG $G$, which on input a challenge $\boldsymbol{\alpha}$, outputs 1 if $\mathbf{E} \boldsymbol{\alpha} \in \operatorname{span}(\mathbf{1})$ and 0 otherwise. From Lemma 2 we know that $\operatorname{Pr}[D=1]$ given that $\boldsymbol{\alpha}$ is uniformly random is $\leq 2 B / P_{\ell}$. On the other hand, the advantage of $D$ is $\operatorname{negl}(k)$, so if $\boldsymbol{\alpha}$ is an output of $G$ then it must be that $D$ outputs 1 with probability at most $2 B / P_{\ell}+\operatorname{negl}(k)$.

We now show indistinguishability of the simulation.

Lemma 4. For every adversary $\mathcal{A}$ who corrupts $P_{R}$, and for every environment $\mathcal{Z}$, it holds that

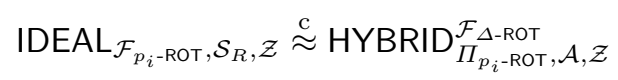

Proof. We first show how $\mathcal{S}_{R}$ (Fig. 5) extracts the corrupt receiver's inputs in step 3c. $\mathcal{S}_{R}$ received the values $u_{i}, u_{i}^{\prime}=u_{i}+e_{i}^{\prime}$ which $\mathcal{A}$ used as input to $\mathcal{F}_{\Delta \text {-ROT }}$. $\mathcal{S}_{R}$ can also compute the actual errors $e_{i}$ (which would equal $e_{i}^{\prime}$ if $P_{R}$ was honest), since it knows $\boldsymbol{r}$ and $\boldsymbol{b}_{i}$. For each $j \in[m+1]$ and $i \in[k], \mathcal{S}_{R}$ defines a value (omitting $j$ subscripts) $q_{i}=q_{i}^{\prime}-\left(u_{i}+s_{i} e_{i}^{\prime}\right)$, and then puts all these values into the vector $\boldsymbol{q}_{j}$. We also compute the values $x_{j}$ and $\boldsymbol{t}_{j}$ as an honest $P_{R}$ would do according to the protocol.

Now, if $P_{R}$ was honest we would have $\boldsymbol{q}_{j}=\boldsymbol{t}_{j}+x_{j} \cdot \boldsymbol{s}$, but it actually holds that

$$
\boldsymbol{q}_{j}=\boldsymbol{t}_{j}+x_{j} \cdot \boldsymbol{s}+\boldsymbol{e}_{j} * \boldsymbol{s}
$$

where $\boldsymbol{e}_{j}$ contains the values $\left(e_{1}-e_{1}^{\prime}, \ldots, e_{k}-e_{k}^{\prime}\right)$ from iteration $j$ of this phase, and $*$ denotes component-wise product. Note that since $e_{i} \in\{0, \pm 1\}$ and $e_{i}^{\prime} \in$ $\{0, \ldots, B-1\}$ we have $\left\|\boldsymbol{e}_{j}\right\|_{\infty} \leq B$ for all $j$.

At this point, although we have computed values $x_{j}$ which could be used to define the inputs of $P_{R}$, these may not be the correct inputs $\mathcal{S}_{R}$ should send to $\mathcal{F}_{p_{i} \text {-ROT }}$. This is because $\mathcal{A}$ could choose, for instance, $\boldsymbol{e}_{j}=\mathbf{1}$, and then the actual inputs would correspond to $x_{j}+1$ and not $x_{j}$. Proposition 1 shows how we obtain the correct inputs. Let $\operatorname{view}(\mathcal{A})$ denote the view of the corrupt receiver at this point in the execution.

Proposition 1. Suppose the consistency check passes, and $\mathcal{A}$ makes no (guess)

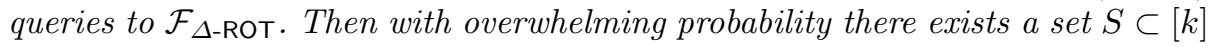
and values $x_{j}^{\prime}, \boldsymbol{e}_{j}^{\prime}$, for $j \in[m]$ such that:

1. $\boldsymbol{q}_{j}=\boldsymbol{t}_{j}+x_{j}^{\prime} \cdot \boldsymbol{s}+\boldsymbol{e}_{j}^{\prime} * \boldsymbol{s}$.

2. For all $i \in[k] \backslash S, \boldsymbol{e}_{j}^{\prime}[i]=0$

3. $H_{\infty}\left(\left(s_{i}\right)_{i \in S} \mid \operatorname{view}(\mathcal{A})\right)=0$

4. $H_{\infty}\left(\left(s_{i}\right)_{i \in[k] \backslash S} \mid \operatorname{view}(\mathcal{A})\right)=k-|S|$. 
Proof. Recall that $\tilde{\boldsymbol{t}}, \tilde{x}$ are the values received by $\mathcal{S}_{R}$ from $P_{R}$ during the consistency check, and we have $\boldsymbol{q}_{j}=\boldsymbol{t}_{j}+x_{j} \cdot \boldsymbol{s}+\boldsymbol{e}_{j} * \boldsymbol{s}$.

This means we can write

$$
\mathbf{Q}=\mathbf{T}+\underbrace{\left(x_{1} \cdot \mathbf{1}+\boldsymbol{e}_{1}\|\cdots\| x_{m} \cdot \mathbf{1}+\boldsymbol{e}_{m}\right)}_{=\mathbf{Y}} * \boldsymbol{s}
$$

where we extend the $*$ operator to apply to every column of $\mathbf{Y}$ in turn.

Define the vectors, in $\mathbb{Z}_{P_{\ell}}^{k}$,

$$
\boldsymbol{\delta}_{x}=\mathbf{1} \tilde{x}-\mathbf{Y} \boldsymbol{\alpha}, \quad \boldsymbol{\delta}_{t}=\mathbf{T} \alpha-\tilde{\boldsymbol{t}}
$$

We can think of these as representing the deviation between what $P_{R}$ sent, and the values $P_{R}$ should have sent, given $\mathbf{Y}, \mathbf{T}$. If the check succeeds, then we know that

$$
\mathbf{Q} \boldsymbol{\alpha}=\tilde{\boldsymbol{t}}+(\mathbf{1} \tilde{x}) * \boldsymbol{s}
$$

and so

$$
\begin{aligned}
(\mathbf{T}+\mathbf{Y} * \boldsymbol{s}) \boldsymbol{\alpha} & =\tilde{\boldsymbol{t}}+(\mathbf{1} \tilde{x}) * \boldsymbol{s} \\
\Leftrightarrow \boldsymbol{\delta}_{t} & =(\mathbf{1} \tilde{x}) * \boldsymbol{s}-(\mathbf{Y} * \boldsymbol{s}) \boldsymbol{\alpha} \\
& =\boldsymbol{\delta}_{x} * \boldsymbol{s}
\end{aligned}
$$

For each index $i \in[k]$, if the check passes then it must hold that either $\boldsymbol{\delta}_{t}[i]=\boldsymbol{\delta}_{x}[i]=0$-which essentially means there was no deviation at position $i$ - or $\boldsymbol{\delta}_{x}[i] \neq 0$. In the latter case, because $s_{i} \in\{0,1\}$, the cheating receiver must guess $s_{i}$ in order to pass the check.

Define $S \subset[k]$ to be the set of all indices $i$ for which $\boldsymbol{\delta}_{x}[i] \neq 0$. From the above, we have that the probability of passing the check (over the random choice of $\boldsymbol{s}$ ) is at most $2^{-|S|}$. If the check passes, this also implies the last two claims of the proposition, that $H_{\infty}\left(\left(s_{i}\right)_{i \in S} \mid \operatorname{view}(\mathcal{A})\right)=0$, and $H_{\infty}\left(\left(s_{i}\right)_{i \in[k] \backslash S} \mid \operatorname{view}(\mathcal{A})\right)=$ $k-|S|$.

Let $\mathbf{Y}_{-S}$ denote the matrix $\mathbf{Y}$ with its rows corresponding to indices in the set $S$ removed. Note that for any $i \notin S$, we have $\boldsymbol{\delta}_{x}[i]=0$ and so $(\mathbf{Y} \boldsymbol{\alpha})_{-S}=$ $\left(\mathbf{Y}_{-S}\right) \boldsymbol{\alpha}=\mathbf{1} \tilde{x}$, which lies in $\operatorname{span}(\mathbf{1})$. Since column $j$ of $\mathbf{Y}$ is equal to $\mathbf{1} x_{j}+\boldsymbol{e}_{j}$ it must also hold that $\left(\mathbf{E}_{-S}\right) \boldsymbol{\alpha} \in \operatorname{span}(\mathbf{1})$, where $\mathbf{E}=\left(\boldsymbol{e}_{1}\|\cdots\| \boldsymbol{e}_{m+1}\right)$.

Applying Lemma 3 with $\mathbf{E}_{-S}$, it then holds that every column of $\mathbf{E}_{-S}$ is in $\operatorname{span}(\mathbf{1})$ with overwhelming probability, provided $2 B / P_{\ell}$ is negligible. Therefore, for every $j \in[m]$, we can compute $x_{j}^{\prime}$ and $\boldsymbol{e}_{j}^{\prime}$ such that the $j$-th column of $\mathbf{Y}$ satisfies $\boldsymbol{y}_{j}=x_{j}^{\prime} \cdot \mathbf{1}+\boldsymbol{e}_{j}^{\prime}$, where $\left(\boldsymbol{e}_{j}^{\prime}\right)_{-S}=0$. These are values needed to satisfy points 1 and 2 .

The set $S$ in the proposition represents the indices where $P_{R}$ cheated, corresponding to the set of bits of $s$ which $P_{R}$ must guess to pass the consistency check. Passing the check also guarantees that the error vectors $\boldsymbol{e}_{j}^{\prime}$ can only be non-zero in the positions corresponding to $S$, which is crucial for the next stage. 
After extracting the corrupt receiver's inputs, we need to show that the random oracle calls made by $\mathcal{Z}$ cannot allow it to distinguish. In particular, if $\mathcal{Z}$ queries

$$
\left.\left(i, \rho,\left(\boldsymbol{q}_{j}-y_{j} \boldsymbol{s}\right) \bmod p_{i}\right)\right)
$$

for some $y_{j} \neq x_{j}^{\prime}$ mod $p_{i}$ then $\mathcal{Z}$ will be able to distinguish, since the simulator's response will be random, whereas the response in the real world will be one of the sender's OT outputs.

From Proposition 1, we know that if no (guess) queries were made to $\mathcal{F}_{\Delta \text {-ROT }}$ then there are exactly $k-|S|$ bits of the secret $s$ that are unknown in the view of $\mathcal{A}$, and these correspond to the index set $[k] \backslash S$.

Now, from the first part of the proposition, we can rewrite a 'bad' query of the form given above as

$$
\left(i, \rho,\left(\boldsymbol{t}_{j}+\boldsymbol{e}_{j}^{\prime} * \boldsymbol{s}+\left(x_{j}^{\prime}-y_{j}\right) \boldsymbol{s}\right) \bmod p_{i}\right)
$$

Since $\boldsymbol{t}_{j}$ and $\boldsymbol{e}_{j}^{\prime} * \boldsymbol{s}$ are fixed in the view of $\mathcal{Z}$, it must be the case that coming up with such a query requires knowing all of $s$. This happens with probability at most $\left(p_{i}-1\right) \cdot 2^{|S|-k}$ per query with index $i$. Taking into account the probability of passing the consistency check, we get an overall success probability bounded by $Q \cdot\left(p_{\ell}-1\right) \cdot 2^{-k}$, where $Q$ is the number of random oracle queries,

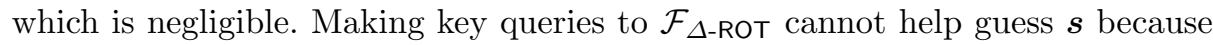
any incorrect guess causes an abort, so this does not affect the distinguishing probability.

\subsection{Choosing the Parameters}

We first show how to securely choose parameters to optimize communication, and then discuss instantiating the key-homomorphic PRF. After the setup phase, and ignoring the short seeds sent in the consistency check, the only communication is to the (chosen) command of $\mathcal{F}_{\Delta \text {-ROT }}$, which can be implemented with $\lambda$ bits of communication when $B=2^{\lambda}$ (see Sect. 4). This gives an overall complexity of $\lambda k m$ bits to generate $m \ell$ random OTs. If $\ell=\omega(\lambda k)$ then we obtain an amortized cost per random OT of $o(1)$, which gets smaller as $\ell$ increases.

To realise 1-out-of-2 bit-OT on chosen strings, each random 1-out-of- $p_{i} \mathrm{OT}$ must be converted to a 1-out-of- $2 \mathrm{OT}$, at a cost of sending $\log p_{i}$ bits from the receiver and 2 bits from the sender (see Appendix A). This adds a cost of $T_{m, \ell}=m \sum_{i=1}^{\ell}\left(\log _{2} p_{i}+2\right)$ bits, and from the prime number theorem we have $p_{i}=O(i \log i)$, so $T_{m, \ell}=m \sum_{i=1}^{\ell} O(\log i)=O(m \ell \log \ell)$, giving an overall, amortized cost of $O(\log \ell)=O(\log k)$ bits per OT when $\ell=\Omega(\lambda k)$.

Instantiating the Key-Homomorphic PRF. We can instantiate $F$ using the random oracle-based construction from Sect. 2 based on learning with rounding, or standard model constructions from LWE [BLMR13,BP14]. With LWR, the parameters affecting security are the dimension $n$ and moduli $p, q$. In our case we 
fix $p=P_{\ell}$ and can choose $n, q$ to ensure security. With an exponential modulus, we know that LWR is at least as hard as LWE with the same dimension $n$ and modulus $q$, where the LWE error distribution is bounded by $\beta=q /\left(2^{\lambda} P_{\ell}\right)$, and $\lambda$ is a statistical security parameter [BPR12, AKPW13]. This gives a modulusto-noise ratio of $q / \beta=O\left(2^{\lambda} P_{\ell}\right)$. LWE with an exponential modulus-to-noise ratio has previously been used to construct attribute-based encryption [GVW13] and fully key-homomorphic encryption [BGG+14], and is believed to be hard if $q / \beta \leq 2^{n^{\epsilon}}$, for some constant $0<\epsilon<1 / 2$ chosen to resist known attacks based on lattice reduction and BKW. To achieve optimal communication in our protocol, we need $\ell=\omega(\lambda k)$, which from the prime number theorem implies that $\log P_{\ell}=\omega(\lambda k \log k)$. This gives $\log (q / \beta)=\omega\left(\lambda^{2} k \log k\right)$, so we can have a dimension of $n=\omega\left(\left(\lambda^{2} k \log k\right)^{1 / \epsilon}\right)$ and ensure security.

\section{Actively Secure Base OTs}

We now show how to implement the functionality $\mathcal{F}_{\Delta \text {-ROT }}^{n, k, \mathbb{G}}$ (Fig. 1), which creates $k$ correlated base OTs over $\mathbb{G}^{n}$, for an additive abelian group $\mathbb{G}$. We achieve active security using a modification of the consistency check from the OT extension protocol by Asharov et al. [ALSZ15, ALSZ17a]. Additionally, in Sect. 4.1 we identify a crucial security flaw in their protocol, whereby a passively corrupted sender can obtain an 'oracle' that allows brute-force guessing of the receiver's choice bits by computing hash values. This bug has since been fixed in a revised version [ALSZ17b], and we apply the same fix to our protocol.

We let $\mathcal{F}_{\text {OT }}^{k, k}$ denote the standard functionality for $k$ sets of 1-out-of-2 OTs on $k$-bit strings. In the correlated OT phase of our protocol, shown in Fig. 6 , the parties first extend the base OTs from $\mathcal{F}_{\text {OT }}$ using a PRF, and the sender $P_{S}$ (who would be receiver when running the main OT extension protocol) then sends the $u_{i}$ values which create the correlation over the group $\mathbb{G}$. The consistency check is based on the check in the OT extension protocol by Asharov et al. [ALSZ15], which is used to verify the sender's inputs are bit strings of the form $\left(b_{i}, b_{i} \oplus \Delta\right)$. We adapt this to ensure they have the form $\left(b_{i}, b_{i}+\Delta\right)$, where $\Delta$ and each $b_{i}$ are vectors of length $n$ over any finite abelian group $\mathbb{G}$. In our protocol the parties then output the correlated base OTs, instead of transposing and hashing them to perform OT extension as in [ALSZ15]. This means we need to account for some leakage on the $s_{i}$ choice bits of $P_{R}$, caused by the consistency check, which is modeled by the key query feature of $\mathcal{F}_{\Delta \text {-ROT }}$.

We also have an additional (non-correlated) Chosen OTs phase, which extends the base OTs further with arbitrary inputs from the sender, $P_{S}$, and the same choice bits from $P_{R}$, in a standard manner using the PRF. Both of these phases can be called repeatedly after the setup phase has run.

\subsection{Security}

We prove the following theorem by considering separately the two cases of a corrupted $P_{R}$ and corrupted $P_{S}$. 


\section{Protocol $\Pi_{\Delta-\mathrm{ROT}}^{n, k, \mathbb{G}}$}

Let $F:\{0,1\}^{k} \times\{0,1\}^{k} \rightarrow \mathbb{G}^{n+k^{\prime}}$ be a PRF and $H: \mathbb{G}^{n+k^{\prime}} \times \mathbb{G}^{n+k^{\prime}} \rightarrow\{0,1\}^{k}$ be a random oracle, where $k^{\prime}$ is chosen so that $|\mathbb{G}|^{k^{\prime}} \geq 2^{k}$.

The protocol consists of two main commands, which can be repeatedly called after the Initialize stage. Both parties maintain a counter $c:=0$.

Initialize: On input (init, $\left.s_{1}, \ldots, s_{k}\right)$ from $P_{R}$ and (init) from $P_{S}$, where $\left(s_{1}, \ldots, s_{k}\right) \in\{0,1\}^{k}:$

1. $P_{S}$ samples seeds $k_{i}^{0}, k_{i}^{1} \leftarrow\{0,1\}^{k}$ for $i \in[k]$

2. The parties run $\mathcal{F}_{\mathrm{OT}}^{k, k}$, where $P_{S}$ is sender with input $\left(k_{i}^{0}, k_{i}^{1}\right)_{i}$ and $P_{R}$ is receiver with input $s_{i}$.

3. $P_{R}$ receives $k_{i}^{s_{i}}$, for $i=1, \ldots, k$.

$\Delta$-OTs: On input (correlated) from $P_{R}$, and (correlated, $\Delta$ ) from $P_{S}$, where $\Delta \in \mathbb{G}^{n}:$

1. Create correlation:

(a) $P_{S}$ samples $\rho \leftarrow \mathbb{G}^{k^{\prime}}$ and sets $\Delta^{\prime}:=(\Delta \| \rho)$.

(b) $P_{S}$ computes $t_{i}^{0}=F\left(k_{i}^{0}, c\right)$ and $t_{i}^{1}=F\left(k_{i}^{1}, c\right)$, then computes

$$
u_{i}=t_{i}^{0}+t_{i}^{1}+\Delta^{\prime}, \quad i=1, \ldots, k
$$

and sends these to $P_{R}$.

(c) $P_{R}$ computes $a_{i}=(-1)^{s_{i}} \cdot F\left(k_{i}^{s_{i}}, c\right)+s_{i} \cdot u_{i} \quad\left(=t_{i}^{0}+s_{i} \cdot \Delta^{\prime}\right)$

(d) Set $c:=c+1$.

2. Consistency Check: For every pair $(\alpha, \beta) \in[k]^{2}$ :

(a) $P_{S}$ computes

$$
\begin{array}{ll}
h_{\alpha, \beta}^{0,0}=H\left(t_{\alpha}^{0}-t_{\beta}^{0}\right), & h_{\alpha, \beta}^{0,1}=H\left(t_{\alpha}^{0}-t_{\beta}^{1}\right) \\
h_{\alpha, \beta}^{1,0}=H\left(t_{\alpha}^{1}-t_{\beta}^{0}\right), & h_{\alpha, \beta}^{1,1}=H\left(t_{\alpha}^{1}-t_{\beta}^{1}\right)
\end{array}
$$

and sends these to $P_{R}$.

(b) $P_{R}$ checks that:
i. $h_{\alpha, \beta}^{s_{\alpha}, s_{\beta}}=H\left(t_{\alpha}^{s_{\alpha}}-t_{\beta}^{s_{\beta}}\right)$
ii. $h_{\alpha, \beta}^{\bar{s}_{\alpha}, \bar{s}_{\beta}}=H\left(u_{\alpha}-u_{\beta}-t_{\alpha}^{s_{\alpha}}+t_{\beta}^{s_{\beta}}\right)$
iii. $u_{\alpha} \neq u_{\beta}$

3. Output: $P_{R}$ outputs the first $n$ components of $a_{i}$, and $P_{S}$ outputs $n$ components of $b_{i}:=t_{i}^{0}$.

Chosen OTs: On input (chosen, $\left.B,\left(u_{i}^{0}, u_{i}^{1}\right)_{i \in[k]}\right)$ from $P_{S}$ and (chosen, $B$ ) from $P_{R}$, where each $u_{i}^{b} \in[0, B-1]$ and $B \leq 2^{k}$ :

1. $P_{S}$ sends $d_{i}^{0}=F\left(k_{i}^{0}, c\right) \oplus u_{i}^{0}$ and $\overline{d_{i}^{1}}=F\left(k_{i}^{1}, c\right) \oplus u_{i}^{1}$ to $P_{R}$, for $i \in[k]$

2. $P_{R}$ outputs $v_{i}=d_{i}^{s_{i}} \oplus F\left(k_{i}^{s_{i}}, c\right)^{a}$

3. Set $c:=c+1$

${ }^{a}$ Only the first $\log _{2} B$ output bits of the PRF are used in this stage.

Fig. 6. Base OT protocol for correlated OTs over an additive abelian group $\mathbb{G}$ 
Theorem 2. If $F$ is a secure PRF, $H$ is a random oracle and $\lambda \leq k / 2$ then protocol $\Pi_{\Delta \text {-ROT }}^{n, k, \mathbb{G}}$ securely realises the functionality $\mathcal{F}_{\Delta \text {-ROT }}^{n, k, \mathbb{G}}$ in the $\overline{\mathcal{F}}_{\mathrm{OT}}^{k, k}$-hybrid model with static security.

Corrupt $\boldsymbol{P}_{\boldsymbol{R}}$. To be secure against a corrupted $P_{R}$, it is vital that $P_{S}$ appends the additional randomness $\rho$ to the input $\Delta$ in step 1a, before creating the correlated OTs. Without this, $P_{R}$ can obtain an 'oracle' that allows guessing whether a candidate value $\tilde{\Delta}$ equals the input of $P_{S}$ or not by just computing one hash value. For example, let $\alpha, \beta$ be indices where $s_{\alpha}=s_{\beta}=0$. Given $t_{\alpha}^{0}, t_{\beta}^{0}, u_{\alpha}$ and the hash values sent by $P_{S}, P_{R}$ can compute $\tilde{t}_{\alpha}^{1}:=u_{\alpha}-t_{\alpha}^{0}-\tilde{\Delta}$, and then test whether $h_{\alpha, \beta}^{1,0}=H\left(\tilde{t}_{\alpha}^{1}-t_{\beta}^{0}\right)$. This only holds if $\Delta=\tilde{\Delta}$, so allows testing any candidate input $\tilde{\Delta}$. Including the extra randomness $\rho$ prevents this attack by ensuring that $\Delta^{\prime}=(\Delta \| \rho)$ always has at least $k$ bits of min-entropy (as long as $|\mathbb{G}|^{k^{\prime}} \geq k$ ), so $P_{R}$ can only guess $\Delta^{\prime}$ with negligible probability ${ }^{5}$.

Note that this step was missing in the published versions of [ALSZ15, ALSZ17a], which leads to an attack on their actively secure OT extension protocol. This has now been fixed in a revised version [ALSZ17b].

To formally prove security against a corrupted $P_{R}$, we construct a simulator $\mathcal{S}_{R}$, who interacts with $\mathcal{F}_{\Delta \text {-ROT }}$ whilst simulating the communication from the honest $P_{S}$ and the $\mathcal{F}_{\text {OT }}$ functionality to the adversary, $\mathcal{A}$. $\mathcal{S}_{R}$ is described below.

1. In the Initialize phase, $\mathcal{S}_{R}$ receives the inputs $\left\{s_{i}\right\}_{i \in[k]}$ from $\mathcal{A}$ to $\mathcal{F}_{\mathrm{OT}}^{k, k}$, then samples random strings $k_{i}^{s_{i}} \leftarrow\{0,1\}^{k}$ and sends these to $\mathcal{A}$.

2. Whenever the $\Delta$-OTs phase is called, $\mathcal{S}_{R}$ starts by sampling $u_{i} \leftarrow \mathbb{G}^{n+k^{\prime}}$, for $i \in[k]$, and sends these to $\mathcal{A}$.

3. In the consistency check, $\mathcal{S}_{R}$ computes and sends the hash values $h_{\alpha, \beta}^{s_{\alpha}, s_{\beta}}=$ $H\left(t_{\alpha}^{s_{\alpha}}-t_{\beta}^{s_{\beta}}\right)$ and $h_{\alpha, \beta}^{\overline{s_{\alpha}}, \overline{s_{\beta}}}=H\left(u_{\alpha}-u_{\beta}-t_{\alpha}^{s_{\alpha}}+t_{\beta}^{s_{\beta}}\right)$. The other two values $h_{\alpha, \beta}^{s_{\alpha}, \overline{s_{\beta}}}, h_{\alpha, \beta}^{\overline{s_{\alpha}}, s_{\beta}}$ are sampled uniformly at random.

4. $\mathcal{S}_{R}$ then sends $\left\{s_{i}\right\}_{i}$ to $\mathcal{F}_{\Delta \text {-ROT }}$, and computes the values $\left\{a_{i}\right\}_{i}$ as an honest $P_{R}$ would in the protocol. $\mathcal{S}_{R}$ then sends $\left\{a_{i}\right\}_{i}$ to $\mathcal{F}_{\Delta \text {-ROT }}$ and increments the counter $c$.

5. Whenever the Chosen OTs phase is called, $\mathcal{S}_{R}$ calls $\mathcal{F}_{\Delta \text {-ROT }}$ with input (chosen, $B$ ), and receives $\left\{v_{i}\right\}_{i=1}^{k} . \mathcal{S}_{R}$ computes $d_{i}^{s_{i}}=F\left(k_{i}^{s_{i}}, c\right) \oplus v_{i}$, samples a random string $d_{i}^{\overline{s_{i}}}$, then sends $d_{i}^{0}, d_{i}^{1}$ to $\mathcal{A}$ and increments $c$.

Lemma 5. If $H$ is a (non-programmable, non-observable) random oracle and $F$ is a secure PRF, then for every adversary $\mathcal{A}$ who corrupts $P_{R}$, and for every environment $\mathcal{Z}$, it holds that

$$
\operatorname{IDEAL}_{\mathcal{F}_{\Delta-\mathrm{ROT}}, \mathcal{S}_{R}, \mathcal{Z}} \stackrel{\mathrm{c}}{\approx} \mathrm{HYBRID}_{\Pi_{\Delta-\mathrm{ROT}}, \mathcal{A}, \mathcal{Z}}^{\mathcal{F}_{\mathrm{OT}}}
$$

${ }_{5}$ This modification is not strictly needed for our application to the protocol in Sect. 3, because $P_{S}$ 's input to $\mathcal{F}_{\Delta \text {-ROT }}$ is always uniformly random and cannot be guessed. However, making this change allows for a simpler, more modular exposition and the functionality may be useful in other applications. 
Proof. We consider a sequence of hybrid distributions, going from the ideal execution to the real execution, defined as follows. The first hybrid $\mathrm{H}_{0}$ is identical to the ideal execution with $\mathcal{S}_{R}$ and $\mathcal{F}_{\Delta \text {-ROT }}$.

Hybrid $\mathrm{H}_{1}$ : This is identical to $\mathrm{H}_{0}$, except that both sets of keys $k_{i}^{0}, k_{i}^{1}$ are sampled by $\mathcal{S}_{R}$, instead of just $k_{i}^{s_{i}}$. We also modify the Chosen OTs phase so that both values $d_{i}^{0}, d_{i}^{1}$ are computed according to the protocol, using the PRF keys and the real inputs of the honest $P_{S}$.

Hybrid $\mathrm{H}_{2}$ : Here we modify $\mathrm{H}_{1}$ further, so that the $u_{i}$ values in the $\Delta$-OTs stage are also computed according to the real protocol, using $P_{S}$ 's real input $\Delta$ and a random value $\rho$. These $u_{i}$ values are then used by $\mathcal{S}_{R}$ to compute the $a_{i}$ 's which are sent to $\mathcal{F}_{\Delta \text {-ROT. }}$

Hybrid $\mathrm{H}_{3}$ : This is the same as $\mathrm{H}_{2}$, except the two hash values $h_{\alpha, \beta}^{s_{\alpha}, \overline{s_{\beta}}}, h_{\alpha, \beta}^{\overline{s_{\alpha}}, s_{\beta}}$ are computed as in the protocol, instead of with random strings.

It is easy to see the view of $\mathcal{Z}$ in $\mathrm{H}_{3}$ is identical to the real execution, since all messages are computed as an honest $P_{S}$ would using the real inputs, and the outputs computed by $\mathcal{F}_{\Delta \text {-ROT }}$ are the same as in the protocol.

Hybrids $\mathrm{H}_{0}$ and $\mathrm{H}_{1}$ are computationally indistinguishable because the keys $k_{i}^{\overline{s_{i}}}$ are unknown to $\mathcal{Z}$, which means the values $d_{i}^{\overline{s_{i}}}$ are indistinguishable from the previously uniform values, by a standard reduction to the PRF security. Similarly, $\mathrm{H}_{1}$ and $\mathrm{H}_{2}$ are computationally indistinguishable because $t_{i}^{\overline{s_{i}}}$ is pseudorandom based on the PRF, so masks $P_{S}$ 's input in $u_{i}$.

Regarding $\mathrm{H}_{2}$, and $\mathrm{H}_{3}$, notice that the two relevant hash values in $\mathrm{H}_{3}$ are equal to

$$
\begin{aligned}
& h_{\alpha, \beta}^{s_{\alpha}, \overline{s_{\beta}}}=H\left(t_{\alpha}^{s_{\alpha}}-t_{\beta}^{\overline{s_{\beta}}}\right)=H\left(t_{\alpha}^{s_{\alpha}}-u_{\beta}+t_{\beta}^{s_{\beta}}+\Delta^{\prime}\right) \\
& h_{\alpha, \beta}^{\overline{s_{\alpha}}, s_{\beta}}=H\left(t_{\alpha}^{\overline{s_{\alpha}}}-t_{\beta}^{s_{\beta}}\right)=H\left(u_{\alpha}-t_{\alpha}^{s_{\alpha}}-\Delta^{\prime}-t_{\beta}^{s_{\beta}}\right)
\end{aligned}
$$

Looking at the values on the right-hand side, $P_{R}$ knows everything in both sets of inputs to $H$ except for $\Delta^{\prime}=(\Delta \| \rho)$.

The only way $\mathcal{Z}$ can distinguish between $\mathrm{H}_{2}$ and $\mathrm{H}_{3}$ is by querying $H$ on one of the two inputs above, which occurs with probability at most $q \cdot|G|^{-k^{\prime}} \leq q \cdot 2^{-k}$, where $q$ is the number of random oracle queries, since $\rho$ is uniformly random in $\mathbb{G}^{k^{\prime}}$ and unknown to $\mathcal{Z}$. This completes the proof.

Corrupt $\boldsymbol{P}_{S}$. When $P_{S}$ is corrupt, the main challenge is to analyse soundness of the consistency check, similarly to [ALSZ15] (with a corrupt receiver in that protocol). Most of the analysis turns out to be identical, so we focus on the differences and state the main properties that we need from that work to show that our protocol securely realises $\mathcal{F}_{\Delta \text {-ROT }}$. For the proof to go through here we need to assume that the statistical security parameter $\lambda$ is no more than $k / 2$, but can always increase $k$ to ensure this holds. 
Note that the main way a corrupt $P_{S}$ may cheat in the protocol is by using different $\Delta^{\prime}$ values when sending the $u_{i}$ values. To account for this, for each $i \in[k]$ we define $\Delta_{i}=u_{i}-t_{i}^{0}-t_{i}^{1}$; if $P_{S}$ behaves honestly then we have $\Delta_{1}=$ $\cdots=\Delta_{k}=\Delta^{\prime}$, otherwise they may be different.

The following lemma is analogous to [ALSZ15, Lemma 3.1], except we work over $\mathbb{G}$ instead of bit strings, and implies that the rest of the analysis of the consistency check from that work also applies in our case. Using the terminology of Asharov et al., if the consistency check passes for some set of messages $\mathcal{T}=$ $\left\{\left\{k_{i}^{0}, k_{i}^{1}, u_{i}\right\}_{i},\left\{H_{\alpha, \beta}\right\}_{\alpha, \beta}\right\}$ and some secret $s$, we say that $\mathcal{T}$ is consistent with $s$. If the check fails then it is inconsistent.

Lemma 6. Let $\mathcal{T}_{\alpha, \beta}=\left\{k_{\alpha}^{0}, k_{\alpha}^{1}, k_{\beta}^{0}, k_{\beta}^{1}, u_{\alpha}, u_{\beta}, H_{\alpha, \beta}\right\}$ and suppose that $H$ is a collision-resistant hash function. Then, except with negligible probability:

1. If $\Delta_{\alpha} \neq \Delta_{\beta}$ and $\mathcal{T}_{\alpha, \beta}$ is consistent with $\left(s_{\alpha}, s_{\beta}\right)$ then $\mathcal{T}_{\alpha, \beta}$ is inconsistent with $\left(\overline{s_{\alpha}}, \overline{s_{\beta}}\right)$.

2. If $\Delta_{\alpha}=\Delta_{\beta}$ and $\mathcal{T}_{\alpha, \beta}$ is consistent with $\left(s_{\alpha}, s_{\beta}\right)$ then $\mathcal{T}_{\alpha, \beta}$ is also consistent with $\left(\overline{s_{\alpha}}, \overline{s_{\beta}}\right)$.

Proof. For the first claim, suppose that $\Delta_{\alpha} \neq \Delta_{\beta}$, and $\mathcal{T}_{\alpha, \beta}$ is consistent with both $\left(s_{\alpha}, s_{\beta}\right)$ and $\left(\overline{s_{\alpha}}, \overline{s_{\beta}}\right)$. Then from the consistency with $\left(s_{\alpha}, s_{\beta}\right)$ we have

$$
h_{\alpha, \beta}^{s_{\alpha}, s_{\beta}}=H\left(t_{\alpha}^{s_{\alpha}}-t_{\beta}^{s_{\beta}}\right), \quad h_{\alpha, \beta}^{\overline{s_{\alpha}}, \overline{s_{\beta}}}=H\left(u_{\alpha}-u_{\beta}-t_{\alpha}^{s_{\alpha}}+t_{\beta}^{s_{\beta}}\right)
$$

On the other hand, consistency with $\left(\overline{s_{\alpha}}, \overline{s_{\beta}}\right)$ implies

$$
h_{\alpha, \beta}^{\overline{s_{\alpha}}, \overline{s_{\beta}}}=H\left(t_{\alpha}^{\overline{s_{\alpha}}}-t_{\beta}^{\overline{s_{\beta}}}\right), \quad h_{\alpha, \beta}^{s_{\alpha}, s_{\beta}}=H\left(u_{\alpha}-u_{\beta}-t_{\alpha}^{\overline{s_{\alpha}}}+t_{\beta}^{\overline{s_{\beta}}}\right)
$$

By the collision resistance of $H$, except with negligible probability it must then hold that

$$
\begin{aligned}
t_{\alpha}^{s_{\alpha}}-t_{\beta}^{s_{\beta}} & =u_{\alpha}-u_{\beta}-t_{\alpha}^{\overline{s_{\alpha}}}+t_{\beta}^{\overline{s_{\beta}}} \\
& =t_{\alpha}^{s_{\alpha}}-t_{\beta}^{s_{\beta}}+\left(\Delta_{\alpha}-\Delta_{\beta}\right)
\end{aligned}
$$

This means $\Delta_{\alpha}=\Delta_{\beta}$, which is a contradiction.

For the second claim, if $\Delta_{\alpha}=\Delta_{\beta}$ then $u_{\alpha}-u_{\beta}=t_{\alpha}^{0}+t_{\alpha}^{1}-t_{\beta}^{0}-t_{\beta}^{1}$, and it can be seen from the above equations that the checks for $\left(s_{\alpha}, s_{\beta}\right)$ and $\left(\overline{s_{\alpha}}, \overline{s_{\beta}}\right)$ are equivalent.

For the case of a corrupted $P_{S}$, we construct a simulator $\mathcal{S}$, who interacts with $\mathcal{A}$ and plays the role of the honest $P_{R}$ and the $\mathcal{F}_{\text {OT }}$ functionality. $\mathcal{S}_{S}$ is described below.

1. $\mathcal{S}_{S}$ receives all the keys $k_{i}^{0}, k_{i}^{1}$ as inputs to $\mathcal{F}_{\mathrm{OT}}$, and then the messages $u_{i}$.

2. Using these it computes $t_{i}^{0}, t_{i}^{1}$ as in the protocol, and $\Delta_{i}=u_{i}-t_{i}^{0}-t_{i}^{1}$. If $P_{S}$ is honest then $\Delta_{1}=\cdots=\Delta_{k}$.

3. $\mathcal{S}_{S}$ defines $\Delta^{\prime}$ to be the most common of the $\Delta_{i}$ 's, and sends the first $n$ components of this as $P_{S}$ 's input to $\mathcal{F}_{\Delta \text {-ROT }}$. 
4. $\mathcal{S}_{S}$ then receives the sets of 4 hash values $H_{\alpha, \beta}=\left\{h_{\alpha, \beta}^{0,0}, h_{\alpha, \beta}^{0,1}, h_{\alpha, \beta}^{1,0}, h_{\alpha, \beta}^{1,1}\right\}$, for each $\alpha, \beta \in[k]$, as part of the consistency check.

5. $\mathcal{S}_{S}$ then uses the transcript of $\mathcal{A}$ to define a set of constraints on the secret $s$ that must be satisfied for the consistency check to pass, by running Algorithm 1 from [ALSZ15]. Note that each of the constraints produced by this algorithm either fixes individual bits of $s$, or the XOR of two bits of $s$, so from this we can efficiently define a set $S(\mathcal{T}) \subset\{0,1\}^{k}$ which describes the set of all $s$ that are consistent with the messages from $\mathcal{A}$.

6. $\mathcal{S}_{S}$ queries $\mathcal{F}_{\Delta \text {-ROT }}$ with (guess, $S(\mathcal{T})$ ). If the query is successful, $\mathcal{S}_{S}$ continues as if the consistency check passed, otherwise, $\mathcal{S}$ aborts.

7. If the check passed, $\mathcal{S}_{S}$ defines values $b_{i}^{\prime}$, for $i \in[k]$ as specified below. These are sent to $\mathcal{F}_{\Delta \text {-ROT. }}$

8. Whenever the Chosen OTs phase is run, $\mathcal{S}_{S}$ uses its knowledge of the keys to extract $P_{S}$ 's inputs $\left(u_{i}^{0}, u_{i}^{1}\right)$, and sends these to $\mathcal{F}_{\Delta \text {-ROT }}$.

The key part of the simulation is step 5, which uses the hash values sent in the consistency check to define the exact bits (or XOR of bits) of the honest $P_{R}$ 's secret $s$ which the corrupt $P_{S}$ tried to guess from cheating. Note that $\mathcal{S}_{S}$ does not define its own secret $s$, as this is already sampled internally in the functionality $\mathcal{F}_{\Delta \text {-ROT. }}$ Therefore, $\mathcal{S}_{S}$ sends a description of all the possible consistent values

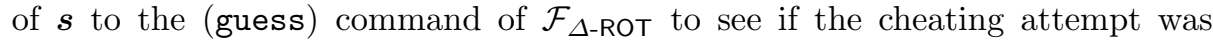
successful or not.

Lemma 7. If $\lambda \leq k / 2$ and $H$ is collision-resistant then for every adversary $\mathcal{A}$ who corrupts $P_{S}$, and for every environment $\mathcal{Z}$, it holds that

$$
\operatorname{IDEAL}_{\mathcal{F}_{\Delta-\mathrm{ROT}}, \mathcal{S}_{S}, \mathcal{Z}} \stackrel{\mathrm{c}}{\approx} \mathrm{HYBRID}_{\Pi_{\Delta-\mathrm{ROT}}, \mathcal{A}, \mathcal{Z}}^{\mathcal{F}_{\mathrm{OT}}}
$$

Proof (sketch): Define the transcript of the simulation up until the consistency check by $\mathcal{T}=\left\{\left\{k_{i}^{0}, k_{i}^{1}, u_{i}\right\}_{i},\left\{H_{\alpha, \beta}\right\}_{\alpha, \beta}\right\}$, and define consistent $(\mathcal{T}, s)$ to be 1 if the consistency check would pass if $s$ is the secret of $P_{R}$, and 0 otherwise. From Algorithm 1 in step 5, recall that the we defined set of all possible secrets $s \in$ $\{0,1\}^{k}$ of an honest $P_{R}$ for which the check would pass to be $S(\mathcal{T})=\{s \in$ $\{0,1\}^{k}$ : consistent $\left.(\mathcal{T}, \boldsymbol{s})=1\right\}$, where $\mathcal{T}$ is the transcript produced by $\mathcal{A}$. Note that from the definition of $S(\mathcal{T})$, the probability that the consistency check passes is $|S(\mathcal{T})| / 2^{k}$ in both the real and ideal executions. To complete the proof we just need to show how to extract the correct values $b_{i}^{\prime}$ defining $P_{S}$ 's output.

Below we give the key results from [ALSZ15] needed to analyse the consistency check.

Lemma 8. For a given transcript $\mathcal{T}$, let $U$ be the largest set of indices such that for all $i, j \in U, \Delta_{i}=\Delta_{j}$, and let $B=[k] \backslash U$ be the complementary set. We have:

1. If $B>\lambda$ then the probability of passing the consistency check is $\leq 2^{-\lambda}$.

2. If the consistency check passes, then for all $\boldsymbol{s}^{\prime} \in S(\mathcal{T})$, it holds that either the bits $\left\{\boldsymbol{s}_{i}^{\prime}\right\}_{i \in B}$ are fixed, or the bits $\left\{\boldsymbol{s}_{i}^{\prime}\right\}_{i \in U}$ are fixed. 
Proof. The first claim follows from Claim B.3 of [ALSZ15] and Lemma 6. The second can be seen from inspection of the proof of Claim B.4 in the same work.

From the first item, we conclude that $|B| \leq \lambda$, except with negligible probability. We claim that this means we first be in the first case of item 2 in the lemma. If the bits $\left\{\boldsymbol{s}^{\prime}\right\}_{i \in U}$ were fixed then the adversary must have guessed $|U|$ bits of the secret to pass the check, but since $|U| \geq k-\lambda \geq \lambda$, this can only happen with probability $\leq 2^{-\lambda}$.

This implies that (except with negligible probability) the bits of $s$ sampled by $\mathcal{F}_{\Delta \text {-ROT }}$ are fixed at the positions $i \in B$, so $\mathcal{S}$ can define a value $b_{i}^{\prime}=t_{i}^{0}+s_{i}$. $\left(\Delta_{i}-\Delta^{\prime}\right)$ from the fact that $s_{i}$ is fixed, for all $i \in B$. We then have $b_{i}^{\prime}=a_{i}-s_{i} \cdot \Delta$, so this defines the correct output that $\mathcal{S}$ sends to $\mathcal{F}_{\Delta \text {-ROT }}$ in step 7 . Note that for all $i \in U$ we have $\Delta_{i}=\Delta^{\prime}$, so we can just let $b_{i}^{\prime}=t_{i}^{0}$. These outputs are then identically distributed to the outputs of $P_{S}$ in the real protocol, so (accounting for the negligible failure events) the simulation is statistically close.

Acknowledgements. I would like to thank Claudio Orlandi for the observation that an XOR-homomorphic PRG would imply non-interactive OT extension, which inspired this work. I am also grateful to the PKC 2018 reviewers for helpful comments.

This work has been supported by the European Union's Horizon 2020 research and innovation programme under grant agreement No 731583 (SODA), and the Danish Independent Research Council under Grant-ID DFF-6108-00169 (FoCC).

\section{A Conversion to 1-out-of-2 OTs}

The main protocol in Sect. 3 produces a batch of random 1-out-of- $p_{i}$ OTs, for multiple small primes $p_{i}$. If an application requires 1-out-of-2 OTs (as is common) then we can convert each of the 1-out-of- $p_{i}$ OTs to a 1-out-of-2 OT at a cost of $O\left(\log p_{i}\right)$ bits of communication using standard techniques, with active security ${ }^{6}$.

\section{Protocol $\Pi_{\text {Conv }}$}

Sender input: strings $s_{0}, \ldots, s_{N-1}$

Receiver input: choice $b \in\{0,1\}$

1. $P_{S}$ obtains random strings $r_{0}, \ldots, r_{N-1}$ from $\mathcal{F}_{p_{i} \text {-ROT }}$

2. $P_{R}$ obtains a random choice $c \in\{0, \ldots, N-1\}$, and the string $r_{c}$.

3. $P_{R}$ sends $d=c-b \bmod N$ to $P_{S}$

4. $P_{S}$ sends $e_{0}=s_{0} \oplus r_{d}$ and $e_{1}=s_{1} \oplus r_{d+1} \bmod N$

5. $P_{R}$ recovers $s_{b}$ by computing $e_{b} \oplus r_{c}$

Fig. 7. Conversion from random 1-out-of- $N$ OT to chosen 1-out-of-2 OT

${ }^{6}$ It is possible to convert a 1-out-of- $N$ OT into $\log _{2} N$ 1-out-of-2 OTs [NP99, KK13], but this technique would not improve the asymptotic communication cost in our case, and is only passively secure. 
In the protocol, shown in Fig. 7, the receiver first converts the random choice $c$ from the 1-out-of- $N$ OT into its chosen input bit $b$ by sending the difference $d=c-b \bmod N$. The sender, who initially has random strings $r_{0}, \ldots, r_{N-1}$, then uses $r_{d}$ and $r_{d+1} \bmod N$ to one-time-pad encrypt its two input messages. The receiver can recover exactly one of these, corresponding to $r_{c}=r_{d+b}$.

Security of the protocol is straightforward. The only concern is that if the receiver is corrupt, $P_{R}$ might choose an inconsistent value $b \in\{2, \ldots, p-1\}$ instead of a bit, so learns a random string instead of one of the sender's two inputs. However, the fact that a corrupt $P_{R}$ may not learn a valid output does not pose a problem, since in this case, in the security proof the simulator can just send an arbitrary choice bit to the $\mathcal{F}_{\text {OT }}$ functionality and simulate the $e_{0}, e_{1}$ messages from the sender with random strings.

\section{References}

[AKPW13] Alwen, J., Krenn, S., Pietrzak, K., Wichs, D.: Learning with rounding, revisited - new reduction, properties and applications. In: Canetti, R., Garay, J.A. (eds.) CRYPTO 2013. LNCS, vol. 8042, pp. 57-74. Springer, Heidelberg (2013). https://doi.org/10.1007/978-3-642-40041-4_4

[ALSZ13] Asharov, G., Lindell, Y., Schneider, T., Zohner, M.: More efficient oblivious transfer and extensions for faster secure computation. In: Sadeghi, A.-R., Gligor, V.D., Yung, M. (eds.) ACM CCS 2013, pp. 535-548. ACM Press, November 2013

[ALSZ15] Asharov, G., Lindell, Y., Schneider, T., Zohner, M.: More efficient oblivious transfer extensions with security for malicious adversaries. In: Oswald, E., Fischlin, M. (eds.) EUROCRYPT 2015. LNCS, vol. 9056, pp. 673-701. Springer, Heidelberg (2015). https://doi.org/10.1007/978-3-66246800-5_26

[ALSZ17a] Asharov, G., Lindell, Y., Schneider, T., Zohner, M.: More efficient oblivious transfer extensions. J. Cryptol. 30(3), 805-858 (2017)

[ALSZ17b] Asharov, G., Lindell, Y., Schneider, T., Zohner, M.: More efficient oblivious transfer extensions with security for malicious adversaries. Cryptology ePrint Archive: Report 2015/061. Version 20171121:125742 (2017). https://eprint.iacr.org/2015/061

[Bea96] Beaver, D.: Correlated pseudorandomness and the complexity of private computations. In: 28th ACM STOC, pp. 479-488. ACM Press, May 1996

[BFP+15] Banerjee, A., Fuchsbauer, G., Peikert, C., Pietrzak, K., Stevens, S.: Keyhomomorphic constrained pseudorandom functions. In: Dodis, Y., Nielsen, J.B. (eds.) TCC 2015. LNCS, vol. 9015, pp. 31-60. Springer, Heidelberg (2015). https://doi.org/10.1007/978-3-662-46497-7_2

[BGG+14] Boneh, D., Gentry, C., Gorbunov, S., Halevi, S., Nikolaenko, V., Segev, G., Vaikuntanathan, V., Vinayagamurthy, D.: Fully key-homomorphic encryption, arithmetic circuit ABE and compact garbled circuits. In: Nguyen, P.Q., Oswald, E. (eds.) EUROCRYPT 2014. LNCS, vol. 8441, pp. 533-556. Springer, Heidelberg (2014). https://doi.org/10.1007/978-3642-55220-5_30 
[BGI16] Boyle, E., Gilboa, N., Ishai, Y.: Breaking the circuit size barrier for secure computation under DDH. In: Robshaw, M., Katz, J. (eds.) CRYPTO 2016. LNCS, vol. 9814, pp. 509-539. Springer, Heidelberg (2016). https://doi. org/10.1007/978-3-662-53018-4_19

[BGI17] Boyle, E., Gilboa, N., Ishai, Y.: Group-based secure computation: optimizing rounds, communication, and computation. In: Coron, J.-S., Nielsen, J.B. (eds.) EUROCRYPT 2017. LNCS, vol. 10211, pp. 163-193. Springer, Cham (2017). https://doi.org/10.1007/978-3-319-56614-6_6

[BLMR13] Boneh, D., Lewi, K., Montgomery, H., Raghunathan, A.: Key homomorphic PRFs and their applications. In: Canetti, R., Garay, J.A. (eds.) CRYPTO 2013. LNCS, vol. 8042, pp. 410-428. Springer, Heidelberg (2013). https://doi.org/10.1007/978-3-642-40041-4_23

[BMR16] Ball, M., Malkin, T., Rosulek, M.: Garbling gadgets for boolean and arithmetic circuits. In: Weippl, E.R., Katzenbeisser, S., Kruegel, C., Myers, A.C., Halevi, S. (eds.) ACM CCS 2016, pp. 565-577. ACM Press, October 2016

[BP14] Banerjee, A., Peikert, C.: New and improved key-homomorphic pseudorandom functions. In: Garay, J.A., Gennaro, R. (eds.) CRYPTO 2014. LNCS, vol. 8616, pp. 353-370. Springer, Heidelberg (2014). https://doi.org/10. 1007/978-3-662-44371-2_20

[BPR12] Banerjee, A., Peikert, C., Rosen, A.: Pseudorandom functions and lattices. In: Pointcheval, D., Johansson, T. (eds.) EUROCRYPT 2012. LNCS, vol. 7237, pp. 719-737. Springer, Heidelberg (2012). https://doi.org/10.1007/ 978-3-642-29011-4_42

[BV15] Brakerski, Z., Vaikuntanathan, V.: Constrained key-homomorphic PRFs from standard lattice assumptions - or: how to secretly embed a circuit in your PRF. In: Dodis, Y., Nielsen, J.B. (eds.) TCC 2015. LNCS, vol. 9015, pp. 1-30. Springer, Heidelberg (2015). https://doi.org/10.1007/978-3-66246497-7_1

[Can01] Canetti, R.: Universally composable security: a new paradigm for cryptographic protocols. In: 42nd FOCS, pp. 136-145. IEEE Computer Society Press, October 2001

[DHRW16] Dodis, Y., Halevi, S., Rothblum, R.D., Wichs, D.: Spooky encryption and its applications. In: Robshaw, M., Katz, J. (eds.) CRYPTO 2016. LNCS, vol. 9816, pp. 93-122. Springer, Heidelberg (2016). https://doi.org/10. 1007/978-3-662-53015-3_4

[GMW86] Goldreich, O., Micali, S., Wigderson, A.: Proofs that yield nothing but their validity and a methodology of cryptographic protocol design (extended abstract). In: 27th FOCS, pp. 174-187. IEEE Computer Society Press, October 1986

[GMW87] Goldreich, O., Micali, S., Wigderson, A.: How to play any mental game or A completeness theorem for protocols with honest majority. In: Aho, A. (ed.) 19th ACM STOC, pp. 218-229. ACM Press, May 1987

[Gol04] Goldreich, O.: The Foundations of Cryptography - Basic Applications, vol. 2. Cambridge University Press, Cambridge (2004)

[GVW13] Gorbunov, S., Vaikuntanathan, V., Wee, H.: Attribute-based encryption for circuits. In: Boneh, D., Roughgarden, T., Feigenbaum, J. (eds.) 45th ACM STOC, pp. 545-554. ACM Press, June 2013 
[HIJ+16] Halevi, S., Ishai, Y., Jain, A., Kushilevitz, E., Rabin, T.: Secure multiparty computation with general interaction patterns. In: Sudan, M. (ed.) ITCS 2016, pp. 157-168. ACM, January 2016

[HIKN08] Harnik, D., Ishai, Y., Kushilevitz, E., Nielsen, J.B.: OT-combiners via secure computation. In: Canetti, R. (ed.) TCC 2008. LNCS, vol. 4948, pp. 393-411. Springer, Heidelberg (2008). https://doi.org/10.1007/978-3540-78524-8_22

[HW15] Hubacek, P., Wichs, D.: On the communication complexity of secure function evaluation with long output. In: Roughgarden, T. (ed.) ITCS 2015, pp. 163-172. ACM, January 2015

[IKNP03] Ishai, Y., Kilian, J., Nissim, K., Petrank, E.: Extending oblivious transfers efficiently. In: Boneh, D. (ed.) CRYPTO 2003. LNCS, vol. 2729, pp. 145-161. Springer, Heidelberg (2003). https://doi.org/10.1007/978-3-54045146-4_9

[IKOS07] Ishai, Y., Kushilevitz, E., Ostrovsky, R., Sahai, A.: Zero-knowledge from secure multiparty computation. In: Johnson, D.S., Feige, U. (eds.) 39th ACM STOC, pp. 21-30. ACM Press, June 2007

[IKOS08] Ishai, Y., Kushilevitz, E., Ostrovsky, R., Sahai, A.: Cryptography with constant computational overhead. In: Ladner, R.E., Dwork, C. (eds.) 40th ACM STOC, pp. 433-442. ACM Press, May 2008

[IKOS09] Ishai, Y., Kushilevitz, E., Ostrovsky, R., Sahai, A.: Extracting correlations. In: 50th FOCS, pp. 261-270. IEEE Computer Society Press, October 2009

[IPS08] Ishai, Y., Prabhakaran, M., Sahai, A.: Founding cryptography on oblivious transfer - efficiently. In: Wagner, D. (ed.) CRYPTO 2008. LNCS, vol. 5157, pp. 572-591. Springer, Heidelberg (2008). https://doi.org/10.1007/978-3540-85174-5_32

[IR89] Impagliazzo, R., Rudich, S.: Limits on the provable consequences of oneway permutations. In: 21st ACM STOC, pp. 44-61. ACM Press, May 1989

[KK13] Kolesnikov, V., Kumaresan, R.: Improved OT extension for transferring short secrets. In: Canetti, R., Garay, J.A. (eds.) CRYPTO 2013. LNCS, vol. 8043, pp. 54-70. Springer, Heidelberg (2013). https://doi.org/10.1007/ 978-3-642-40084-1_4

[KKRT16] Kolesnikov, V., Kumaresan, R., Rosulek, M., Trieu, N.: Efficient batched oblivious PRF with applications to private set intersection. In: Weippl, E.R., Katzenbeisser, S., Kruegel, C., Myers, A.C., Halevi, S. (eds.) ACM CCS 2016, pp. 818-829. ACM Press, October 2016

[KOS15] Keller, M., Orsini, E., Scholl, P.: Actively secure OT extension with optimal overhead. In: Gennaro, R., Robshaw, M. (eds.) CRYPTO 2015. LNCS, vol. 9215, pp. 724-741. Springer, Heidelberg (2015). https://doi.org/10.1007/ 978-3-662-47989-6_35

[NNOB12] Nielsen, J.B., Nordholt, P.S., Orlandi, C., Burra, S.S.: A new approach to practical active-secure two-party computation. In: Safavi-Naini, R., Canetti, R. (eds.) CRYPTO 2012. LNCS, vol. 7417, pp. 681-700. Springer, Heidelberg (2012). https://doi.org/10.1007/978-3-642-32009-5_40

[NP99] Naor, M., Pinkas, B.: Oblivious transfer and polynomial evaluation. In: 31st ACM STOC, pp. 245-254. ACM Press, May 1999

[OOS17] Orrù, M., Orsini, E., Scholl, P.: Actively secure 1-out-of- $N$ OT extension with application to private set intersection. In: Handschuh, H. (ed.) CTRSA 2017. LNCS, vol. 10159, pp. 381-396. Springer, Cham (2017). https:// doi.org/10.1007/978-3-319-52153-4_22 
[PSS17] Patra, A., Sarkar, P., Suresh, A.: Fast actively secure OT extension for short secrets. In: NDSS 2017. Internet Society (2017)

[PSZ18] Pinkas, B., Schneider, T., Zohner, M.: Scalable private set intersection based on OT extension. ACM Trans. Priv. Secur. 21(2), 7:1-7:35 (2018)

[Yao86] Yao, A.C.-C.: How to generate and exchange secrets (extended abstract). In: 27th FOCS, pp. 162-167. IEEE Computer Society Press, October 1986 